\title{
Spillover Effects In Seeded Word-Of-Mouth Marketing Campaigns
}

\author{
Inyoung Chae \\ $\mathrm{PhD}$ Candidate in Marketing \\ INSEAD \\ Boulevard de Constance, Fontainebleau, France \\ Email. inyoung.chae@insead.edu \\ Andrew T. Stephen \\ L'Oréal Professor of Marketing \\ University of Oxford, Saïd Business School \\ Park End Street, Oxford, United Kingdom \\ Email. Andrew.Stephen@sbs.ox.ac.uk
}

\begin{abstract}
Yakov Bart
Assistant Professor of Marketing

Northeastern University, D'Amore-McKim School of Business

360 Huntington Avenue, Boston MA, USA

Email.y.bart@neu.edu
\end{abstract}

\author{
Dai Yao \\ Assistant Professor of Marketing \\ National University of Singapore, NUS Business School \\ 15 Kent Ridge Drive, Singapore \\ Email.dai.yao@nus.edu.sg
}

Forthcoming, Marketing Science

Inyoung Chae (inyoung.chae@ insead.edu) is a PhD candidate in marketing at INSEAD, Andrew T. Stephen (Andrew.Stephen@ sbs.ox.ac.uk) is the L'Oréal Professor of Marketing at the Saïd Business School, University of Oxford, Yakov Bart (y.bart@neu.edu) is assistant professor of marketing at the D'Amore-McKim School of Business, Northeastern University, and Dai Yao (dai.yao@nus.edu.sg) is assistant professor of marketing at NUS Business School, National University of Singapore. The authors thank Andy Gershoff, and seminar participants at the University of Texas, Austin and Northeastern University for their helpful comments. This research was supported by the INSEAD Alumni Fund and the Marketing Science Institute (grant \#4-1935). 


\title{
Spillover Effects In Seeded Word-Of-Mouth Marketing Campaigns
}

\begin{abstract}
Seeded marketing campaigns (SMCs) involve firms sending product samples to selected customers and encouraging them to spread word of mouth (WOM). Prior research has examined certain aspects of this increasingly popular form of marketing communication, such as seeding strategies and their immediate efficacy. Building on prior research, this study investigates the effects of SMCs that extend beyond the generation of WOM for a campaign's focal product by considering how seeding can affect WOM spillover effects at the brand and category levels. The authors introduce a framework of SMC-related spillover effects, and then empirically estimate these with a unique dataset covering 390 SMCs for products from 192 different cosmetics brands. Multiple spillover effects are found, suggesting that while SMCs can indeed be used primarily to stimulate WOM for a focal product, marketers must also account for brand- and category-level WOM spillover effects. Specifically, product seeding increases conversations about that product among non-seed consumers, and, interestingly, decreases WOM about other products from the same brand and about competitors' products in the same category as the focal product. These findings indicate that marketers can use SMCs to focus online WOM on a particular product by drawing consumers away from talking about other related, but off-topic, products.
\end{abstract}

Keywords: Word-of-Mouth, Seeded Marketing Campaigns, Social Media, Spillover Effects, Viral Marketing. 


\section{Introduction}

Consumer-to-consumer word-of-mouth (WOM) communications are widely believed to have a powerful influence on consumer behavior. Previous studies have shown that WOM shapes consumer expectations and pre-usage attitudes (Anderson 2003a; Herr, Kardes and Kim 1991), affects choice and purchase decisions across a variety of product categories (Arndt 1967;

Berger and Schwartz 2011; Chevalier and Mayzlin 2006; Godes and Mayzlin 2004; Whyte 1954), changes post-usage perceptions of products (Bone 1995), and improves customer acquisition, retention, and sales (Kumar, Petersen, and Leone 2010; Lamberton and Stephen 2015; Libai et al. 2010; Sonnier, McAlister, and Rutz 2011; Stephen 2016; Stephen and Galak 2012; Trusov, Bucklin, and Pauwels 2009; Villanueva, Yoo, and Hanssens 2008). Further, with the increasingly widespread use of social media, including online discussion forums and online review platforms, firms have taken a greater interest in finding ways to generate and leverage consumer-toconsumer WOM to help achieve their marketing objectives.

This paper considers a popular approach firms use for generating product-related WOM: seeded marketing campaigns (SMCs). Sometimes called a buzz, influencer, sampling, or viral marketing campaign, a typical SMC involves a firm seeding a focal product with selected consumers (e.g., by sending them samples) and asking those consumers to generate WOM about that product. Although WOM can take many forms and thus occur in a large number of contexts, it is increasingly common for firms to ask seeded consumers to generate WOM about the focal product in the form of posts in online forums or on social media websites, or as reviews on retail websites. SMCs are popular among firms of all sizes. For example, a recent industry study by the American Marketing Association and the Word of Mouth Marketing Association reports that one-third of marketers either have run or plan to run campaigns in which samples are seeded 
with consumers, and three-quarters either have used or plan to use consumers to start spreading WOM (WOMMA 2014). This form of firm-encouraged, consumer-to-consumer WOM has been referred to as "amplified" WOM and is distinct from "organic" WOM, which occurs naturally without direct firm involvement (Libai et al. 2010).

Prior research on amplified WOM marketing programs in general and, more specifically, SMCs focuses predominantly on questions related to brand characteristics (i.e., what brands are more likely to generate WOM or information sharing), target characteristics (i.e., whom should firms select as seeds), incentives (i.e., how can seeds be implicitly and explicitly encouraged), and marketing outcomes (i.e., what types of consumer behaviors are influenced). For example, Lovett, Peres, and Shachar (2013) study talked-about brands and connect their characteristics to social, emotional, and functional drivers of WOM in both offline and online settings. Hinz et al. (2011) examine various strategies for selecting seeds and find that the best seeds are often consumers with high levels of social connectivity. Godes and Mayzlin (2009) find that the most loyal existing customers might not be ideal seeds because their friends (to whom they would transmit WOM) are likely to also be customers. Libai, Muller, and Peres (2013) show how WOM outcomes combine acceleration and expansion components to generate value. Lastly, a rich literature studies incentive mechanisms for generating WOM-based customer referrals (e.g., Biyalogorsky, Gerstner, and Libai 2001; Kornish and Li 2010; Ryu and Feick 2007; Stephen and Lehmann 2015), and Schmitt, Skiera, and Van den Bulte (2011) examine the value of customers acquired through WOM referral programs.

Despite the rich literature on WOM marketing and SMC-related topics, prior research tends to focus on either the immediate effects of SMCs on WOM transmissions for a campaign's focal product, or how best to design an SMC (e.g., selecting "optimal" seeds). Critically, the broader consequences of running an SMC have received scant attention. Given that it is well 
established that WOM about a focal product can be triggered or amplified by seeded conversations, it is conceivable that WOM on products related to but different from a focal product might also be affected by firms' seeding actions. In other words, seeding a focal product might trigger certain spillover effects. For example, an SMC for Chanel lipstick in which seed consumers receive a sample product and post reviews about that product in an online community might affect the amount of WOM generated in that community about other Chanel products (i.e., spillover effects with respect to WOM about the same brand's products in other categories) and/or about competing products (i.e., spillover effects with respect to WOM about other brands' products in the same category). In addition to brand- and category-related spillover effects, WOM about a focal product among consumers in one segment could spill over to affect WOM among consumers in other segments. For example, although an SMC for Chanel lipstick might target specialist or expert cosmetics users (and select seeds from this segment), WOM generated by members of this segment could also influence WOM among consumers in other segments, such as more generalist or novice cosmetics users.

Although seeding has gained recognition as a key marketing communication tool, research on the types of WOM spillovers described here is limited. Two streams of prior research do, however, suggest in general that WOM spillovers (e.g., brand and category spillovers) are possible. First, diffusion research considers models with within- and cross-brand influence on new product diffusion processes (Libai, Muller, and Peres 2009), WOM externalities (Peres and Van den Bulte 2014), and indirect effects on brand-level diffusion due to category-level sales (Krishnan, Seetharaman, and Vakratsas 2012). Second, qualitative research by Kozinets et al. (2010) finds that firm-initiated attempts to generate WOM in online communities by targeting prominent bloggers can also affect online conversations among the general population. This suggests that WOM among one consumer segment can spill over into other consumer segments 
(e.g., from "expert" bloggers to the general population). Taken together, these streams of research suggest qualitatively and theoretically that WOM spillovers from SMCs exist.

Building on these findings, the current research examines how SMCs trigger a comprehensive set of WOM spillover effects with respect to a focal product, products from the same brand but in different categories, and competing products from different brands in the same category. The latter two spillover effects represent indirect, probably unintended, and potentially unfavorable consequences of a firm's decision to use an SMC for a focal product. We consider two research questions: (1) What types of WOM spillover effects are triggered by seeding? And (2) What is the nature of these effects (positive or negative) and how large are they? To address these questions, we empirically identify multiple spillover effects using a unique dataset of 390 SMCs for products across 192 different cosmetics brands. This research fills an important gap in the literature on SMCs and WOM by highlighting and examining the possibility that conversations triggered by seeding a focal product can affect conversations about related but, different products from either the same brand as the focal product or competitors. Despite their potential importance, such effects have not been studied in prior SMC-related research.

\section{Types of Word Of Mouth Spillover Effects}

Before presenting our conceptual framework, we introduce a typology of WOM spillover effects that could be triggered by firms' SMC-initiating seeding actions. For the purposes of this typology and the subsequent conceptual framework, we distinguish between two types of WOM based on the consumer source: seed and non-seed. Seed WOM is generated by a campaign's seed consumers; i.e., those who are typically sent product samples, and expected to initiate 
conversations by reviewing them. Non-seed WOM is generated by all other consumers; i.e., those who are not selected as seeds.

The distinction between seed and non-seed WOM is important because we define WOM spillover effects as the positive or negative influence of seed WOM about an SMC's focal product on non-seed WOM about the focal product, the corresponding brand, or other products or brands in the category. ${ }^{1}$ As this definition suggests, we consider three types of WOM spillover effects that can arise from an SMC for a focal product:

1. Focal product spillovers. Change in amount of WOM generated by non-seed consumers about the focal product. For example, in an SMC for Chanel lipstick, a focal product spillover would be a change in the amount of non-seed WOM about Chanel lipstick;

2. Brand spillovers. Change in amount of WOM generated by non-seed consumers about products from the same brand as the focal product, but not about the focal product. I.e., WOM is "within brand" and "across category." For example, in an SMC for Chanel lipstick, a brand spillover would be a change in the amount of non-seed WOM about non-lipstick products from Chanel, such as Chanel nail polish;

3. Category spillovers. Change in amount of WOM generated by non-seed consumers about products from the same category as the focal product, but not about the focal product or its brand. I.e., WOM is "within category" and "across brand." For example, in an SMC for Chanel lipstick, a category spillover would be a change in the amount of non-seed WOM about non-Chanel products in the lipstick category, such as Revlon lipstick.

\footnotetext{
${ }^{1}$ Seed and non-seed WOM in this context is WOM activity that is directly or indirectly triggered by the SMC. Of course, so-called "organic" WOM that is not a consequence of SMC-related actions by firms can coexist. Although not theoretically the focus of this research, as described later, we do attempt to empirically control for this.
} 
These spillover effects can be positive or negative. Positive spillover effects in our framework are such that an increase in seed WOM about a focal product results in an increased amount of non-seed WOM about the focal product, other-category products from the same brand, or same-category products from different brands for focal product, brand, and category spillovers, respectively. Conversely, negative spillover effects occur if increased seed WOM about a focal product results in decreased amounts of non-seed WOM of these types.

Further, each of these WOM spillover effects can occur either within or between consumer segments. Within-segment spillovers occur among consumers who are in the same segment as the seed consumers. Between-segment spillovers are, on the other hand, those in which consumers in a different segment to the seeds are influenced, leading to changes in the amounts of WOM they generate. For example, if a firm selects seed consumers who are heavy category users (as is often the case), within-segment spillover effects would involve a change (positive or negative) in the amount of non-seed WOM by consumers who are also heavy users of products in that category. Conversely, between-segment spillover effects would involve a change in the amount of non-seed WOM by consumers who are not heavy users in that category.

For the purposes of our empirical analysis, we consider a setting with two segments, which we refer to as specialists (e.g., experts, heavy users, early adopters) and generalists (i.e., everyone else). Firms usually select seeds from a specialist segment (WOMMA 2005), ${ }^{2}$ and this is also the case in our empirical setting. ${ }^{3}$ Thus, within-segment spillovers are those where seed WOM affects non-seed WOM among specialists, and between-segment spillovers are those where seed WOM affects non-seed WOM among generalists. Note, in the case of betweensegment spillovers, that it is plausible that the effect of seed WOM on non-seed WOM will be

\footnotetext{
${ }^{2}$ Firms typically seed with specialists (e.g., advocates, enthusiasts, experts, influentials, innovators, or mavens) because firms believe that specialists are more intrinsically motivated to participate in SMCs by generating WOM, more credible, and more receptive to extrinsic motivators (i.e., incentives) such as free product samples.

${ }^{3}$ To be clear, in our setting seeds must be specialists, and non-seeds can be either specialists or generalists.
} 
indirect, in the sense that seed WOM may first influence non-seed WOM among specialists (within-segment), which will then influence non-seed WOM among generalists (betweensegment), consistent with two-step flows of communications (Katz and Lazarsfeld 1955) and cross-segment diffusion (e.g., Rogers 1995; Van den Bulte and Joshi 2007). While our conceptualization in the next section primarily focuses on focal product, brand, and category spillover effects in general and does not specifically distinguish between within- and betweensegment effects, for completeness and because our data allow for it, we incorporate these effects in the empirical analysis reported later.

\section{Conceptual Framework}

\subsection{Focal Product Spillover Effect}

Marketers have long recognized that consumers often share and spread product-related information over social ties (e.g., see Van den Bulte and Wuyts 2007 and Hinz et al. 2011 for reviews of social contagion and seed/viral marketing). We expect that when seed consumers spread focal product WOM, the non-seed consumers exposed to this information will become more likely to generate non-seed WOM about the focal product - a positive focal product spillover effect. For the most part, this is a primary goal of an SMC; i.e., the seeds influencing non-seeds to generate WOM (or "buzz") about the focal product.

This positive focal product spillover effect is expected to occur for a number of related reasons. First, non-seed consumers may be prompted to talk about the focal product because the seed WOM piques their interest, and non-seeds use their own WOM to discuss what is 
essentially a new and uncertain product ${ }^{4}$ so that they can obtain additional information to resolve their uncertainty. This effect is not specific to SMCs and is likely to follow any firm-initiated communication about a new product. Second, consistent with Watts and Dodds (2007), nonseeds exposed to seed WOM about a focal product may be susceptible to influence and thus made more likely to generate WOM themselves. Third, when exposed to seed WOM about a focal product, non-seeds (particularly specialists) might feel a need to contribute their own opinions to the burgeoning conversation for self-presentation or self-enhancement reasons, such as signaling expertise or reputation (e.g., Wojnicki and Godes 2008). Finally, the notion that seed WOM on a particular topic (in this case, the focal product) will result in more non-seed WOM on the same topic is consistent with the two-step flow of communications model (Katz and Lazarsfeld 1955), as well as research on two-stage diffusion models where new product adoptions spread from innovators to imitators (Rogers 1995; Van den Bulte and Joshi 2007). Stated formally:

H1: Seed WOM about an SMC's focal product will lead to increased non-seed WOM about the focal product (i.e., positive focal product spillover effect).

\subsection{Brand and Category Spillover Effects}

The potential for marketing activity for a focal product to affect consumers' attitudes and actions related to other products from the same brand as the focal product (i.e., brand spillover effects) and products under different brands from the same category as the focal product (i.e., category spillover effects) is generally supported by prior research, although typically in contexts unrelated to WOM or SMCs. For example, Balachander and Ghose (2003) find reciprocal

\footnotetext{
${ }^{4}$ Most focal products in SMCs tend to be new and experience goods (e.g., new books, videogames, cosmetics), hence they are likely to be associated with higher levels of uncertainty.
} 
spillover effects from brand extensions, Libai et al. (2009) identify significant cross-brand adoption effects, and Lewis and Nguyen (2015) find that online display advertisements affect online search activity for competitors' brands. The literature on umbrella branding strategies also considers spillover effects. For instance, Erdem and Sun (2002) empirically show how changes in such marketing-mix instruments as advertising, coupon availability, and in-store promotions for a focal product affect consumer choice for both the focal product and for products under the same brand but in a different category (i.e., a brand spillover effect). Spillover effects are also important in the brand alliance literature. Simonin and Ruth (1998), for example, show that when two brands form an alliance, consumers' pre-alliance attitudes toward one brand are positively related to consumers' post-alliance attitudes toward both that brand and the other brand.

Research on marketing spillover effects across various non-WOM contexts tends to find positive spillover effects such that a marketing-related action (e.g., placing advertisements, reducing a price, or forming an alliance) has an effect in the same direction on both a focal product and associated products. For instance, a positive (brand) spillover effect along the lines of those described by Erdem and Sun (2002) would be where traditional advertising for Colgate toothpaste lifts sales for Colgate toothpaste and Colgate mouthwash (i.e., same brand but different category). Similarly, in the context of online advertising, Lewis and Nguyen (2015) find positive (category) spillover effects where, for example, display ads for Samsung tablets increase search volume for both that product and Apple iPads. Positive (category) spillover effects of advertising on sales have also been identified in the contexts of apparel advertising through direct mail (Anderson and Simester 2013) and restaurant advertising with online ads (Sahni 2013). Positive (brand) spillover effects are also found in the context of celebrity endorsements. For example, Garthwaite (2014) shows book endorsements by Oprah Winfrey lead to higher sales for both endorsed titles and non-endorsed titles by the same author. Finally, another 
example of a positive (category) spillover effect, although with unfavorable outcomes instead of favorable ones, is the "perverse halo effect" identified by Borah and Tellis (2015) where negative consumer sentiment toward an automotive brand due to a product recall increases negative sentiment toward other automotive brands in the same category (e.g., a recall for Toyota minivans can increase negative sentiment toward both Toyota minivans and Honda minivans).

An explanation for positive spillover effects of this nature is that firms' marketing efforts for a specific topic or focal product can cue consumers to think about associated but broader concepts related to non-focal products that are either from the same brand but in a different category, or in the same category but from a different brand. In other words, thinking about a focal product could trigger thoughts about higher-level concepts (brand, category), which in turn open up the possibility of thinking about other products (Berger and Schwartz 2011). This mechanism is consistent with Construal Level Theory (Liberman and Trope 1998; Trope, Liberman, and Wakslak 2007; Trope and Liberman 2010; see also Dhar and Kim 2007; Fiedler 2007). In line with this theory, a positive spillover effect could occur if consumers, in response to exposure to a stimulus, adopt an abstract and broad perspective (higher-level construal). When prompted by marketing actions about a focal product, consumers could expand their thoughts to encompass brand- or category-level concepts.

On the other hand, the opposite is also possible; i.e., negative spillover effects. This could occur if consumers, in response to exposure to a stimulus that is about a focal product, adopt a concrete and narrow perspective (lower-level construal). That is, when prompted by marketing actions or other consumers' WOM for a focal product they may concentrate on specific aspects of the focal product, such as particular product-specific attributes or functionalities, and adoption of such concrete and narrow perspective may then suppress the retrieval of thoughts related to more abstract brand- and category-level concepts that might have otherwise occurred (Kardes, 
Cronley, and Kim 2006). If this is the case, we should see negative brand and/or category spillover effects. In other words, activation of narrower, concrete mental representations of a focal product could suppress retrieval of broader, abstract representations of related, but not focal, products. This mechanism has been extensively examined in prior literature on memory and cognitive processing, and is related to work explaining how the activation of one set of thoughts can inhibit the activation of related, competing thoughts (e.g., Anderson and Bjork 1994, Anderson and Bell 2001, Anderson 2003b).

We posit that SMCs can trigger negative brand and category spillover effects. This is because we expect that seeds, when writing about the focal product, will adopt a lower-level construal of the focal product (and thus be less likely to adopt a higher-level construal), and that this in turn will affect the nature of the WOM generated by non-seeds by making them also more likely to concentrate more on the focal product. In other words, if seed WOM reflects a lowerlevel, more concrete representation of the focal product, that will "frame the conversation" and make it more likely for non-seeds to follow the specific level of representation that favors the focal product and suppresses retrieval of broader thoughts that could lead to WOM about other products from the same brand or same category.

We expect seeds in SMCs to adopt lower-level construals of focal products for three related reasons. First, a key characteristic of most SMCs is that firms encourage (or in some cases require) seed consumers to write detailed reviews. This should increase the tendency for seeds to focus more on concrete aspects of the product and trial experience and hence talk more about what a focal product can be used for, how to use it, how it works, what features it has, what to expect after use, and how well it works; i.e., considerations about functionality and feasibility, rather than about desirability. These types of thoughts have been shown to reflect lower-level construals (Dhar and Kim 2007; Liberman and Trope 1998). 
Second, the detailed firsthand experience with a focal product that seeds are expected to describe in a typical SMC encourages seed consumers to have close and frequent physical access to a focal product, thus shortening the "sensory distance" between seeds and the focal product and fostering lower-level construals (Kardes et al. 2006, Trope and Liberman 2003).

Finally, seed WOM is typically structured in a very specific narrative format, where a seed consumer uses words and images to describe the sequence of obtaining a product sample, unwrapping it, inspecting it, using it, and examining consequences (e.g., explaining how well it works). This format of exposition is also likely to be consistent with lower-level construal.

In sum, seed WOM of this nature should suppress retrieval of broader thoughts that could lead non-seeds to talk about other products from the same brand or category, and will therefore drive negative brand and/or category spillover effects. Stated formally:

H2: Seed WOM about an SMC's focal product will decrease non-seed WOM about other products from the same brand as the focal product.

H3: Seed WOM about an SMC's focal product will decrease non-seed WOM about products from other brands in the same category as the focal product.

If these hypothesized effects are the result of the above-described mechanism, the effects should be stronger when seeds can more easily relate their experiences in a functional sense, because this more readily lends itself to lower-level construals that focus on attributes, functionality, and performance. However, not all products can be discussed or talked about in such concrete terms. Some product usage experiences when described to others are easy to express in general, holistic terms but hard to formulate more concretely. For example, it might be relatively easy for a consumer to describe a perfume in abstract, more hedonic terms (e.g., "I loved the scent, and it made me feel great"), but much harder to communicate the product in more concrete terms (e.g., "The scent had notes of lavender and fresh-cut grass, with a hint of 
coconut"). On the other hand, it should be fairly easy for consumers to describe a product such as a skin cream for reducing acne blemishes in specific, concrete, functional terms (e.g., "After using the cream twice a day for a week I had noticeably fewer blemishes on my face and less redness"). Moore (2015) provides evidence for this difference in the types of explanations offered by consumers when writing product reviews for different product types. She shows that utilitarian products are more often explained through actions and choices (i.e., lower-level construals are more likely because of specific functionality and feasibility considerations), whereas hedonic products are more often explained through reactions and feelings (i.e., higherlevel construals are more likely because of holistic desirability considerations).

Thus, if negative brand and category spillover effects occur for the reasons explained above, we should expect negative spillover effects to be stronger when the focal product is of a more utilitarian nature because it can be more easily represented in terms of attributes, functionality, and performance. This, in turn, makes it more likely that it will be construed by seeds (and, subsequently, also non-seeds) at a lower level. Stated formally:

H4: Negative (a) brand and (b) category spillover effects will be stronger for products that are more utilitarian in nature.

\section{Data}

\subsection{Overview}

We now turn to empirically testing our hypotheses using a large and unique consumer WOM dataset from one of the largest Internet portal sites in South Korea, Naver, which has almost 80\% market share in South Korea (The Economist 2014). Although Naver has many features (including South Korea's most popular search engine), the feature we focus on is the 
discussion forums and, within that, the product-related forums in which consumers share product-related opinions and reviews. Similar to English-language portals that host online forums (e.g., Yahoo), Naver's forums have tree-like (or threaded) discussion structures such that discussions on related topics are grouped together. Specifically, in the product-related discussion forums, forums are organized around broad product types.

Our dataset covers product-related discussion forums for cosmetics products. We chose this industry as the study's focus for several reasons. First, in Naver, cosmetics are one of the most prominent product types in terms of product-related discussion forum activity and, importantly, firm-initiated SMCs. Second, it is generally common for firms to use SMCs with product sampling to promote cosmetics products. Third, the South Korean cosmetics industry is large (2012 sales of 1.3 trillion Won, or approximately US $\$ 1$ billion) and growing at a 7.7\% compound annual rate (Euromonitor 2013). Fourth, the cosmetics industry has features that allow us to identify both the brand and category spillover effects of interest because it has many brands that offer products across multiple categories (e.g., lipstick, nail polish, basic skin care). Finally, consumers are more likely to search for product reviews for cosmetics due to high levels of product diversity and the fact that cosmetics products are experience goods.

In addition to the standard hierarchical organization of product-related conversations, a unique feature of Naver's forums, which we exploit in order to identify spillover effects separately for specialist and generalist consumer segments, is that cosmetics product-focused forums are divided into two types. The first type, called "Online Café," is a membership-only forum for specialist consumers. Independent community administrators (who are not employees of Naver) regulate membership and require members to demonstrate high levels of engagement through active participation in discussions. For example, in one of the "Online Cafe'" forums in our dataset, members must write at least 20 product-related posts and submit at least three 
product reviews per month to retain their membership. Membership confers posting rights in members-only forums (i.e., non-members of Online Café forums can read but not write posts) and, importantly, makes one eligible to be selected as a seed in firm-initiated SMCs for cosmetics products. Thus, all members of product-related discussion forums of this type are considered to be specialists. The second type of forum, called "Knowledge In," does not have any membership requirements and allows anyone to write posts. Consumers posting on this forum, therefore, are considered to be generalists. These consumers can read posts by specialists in the Online Café forums but not write posts; they can only write posts in their own Knowledge In forums.

\subsection{Seeded Marketing Campaigns for Cosmetics Products on Naver}

Firms use only the specialist forums (i.e., Online Café) for seeding products with specialists. This means that a consumer must be a specialist in order to be selected as a seed in a campaign. The standard SMC procedure followed by all firms on this platform is as follows:

1. The firm initiates an SMC by sending a campaign request to a forum's community administrator, who is an independent "super member" of the forum. The request includes a description of the product, the number of seeds to be recruited (the average number is approximately 25 per SMC), a timeline for the physical delivery of the samples to seeds, and a timeline for when seeds are expected to post product opinions and reviews (i.e., seed WOM) on the campaign's specialist forum.

2. The community administrator posts an announcement on the specialist forum that invites members to apply to be a seed for a particular campaign. 
3. Seeds are selected randomly ${ }^{5}$ from the applicant pool, and samples are shipped.

4. Seeds receive samples and are asked ${ }^{6}$ to test the products and post reviews in the designated SMC section of the forum, typically within 2-3 weeks. Seeds' reviews tend to be very thorough and often include their detailed opinions, descriptions of their experiences with the product, "before and after" photos (e.g., before and after a skincare treatment), and details on how products can be used.

Following our typology of spillover effects in $§ 2$, a review posted by a seed in a specialist forum is considered to be an instance of focal product seed WOM. Then, once seeds begin to post their reviews and opinions, this may affect non-seed WOM-generation activity among both specialists and generalists. The following actions correspond to the spillover effects described earlier:

- $\quad$ Focal product non-seed WOM by specialists. Other, non-seed specialists may contribute posts to the conversation in their own board (seeds' reviews are in a separate board). These can include reviews, recommendations, videos, and photos.

- $\quad$ Focal product non-seed WOM by generalists. Non-members of the specialist forum cannot post in the specialist forum, although—importantly—-they can read posts in the specialist forum. Non-members can, however, post in corresponding productrelated boards in the generalist forum. Thus, they can be exposed to focal product seed and non-seed WOM by specialists (acquiring information from and being influenced by specialists) and then disseminate this information, as well as their own opinions, in the relevant product-related discussions in the generalist forum.

\footnotetext{
${ }^{5}$ Administrators randomly select seeds. They do, however, make small adjustments to ensure that seed opportunities are available approximately equally for all (active) members over time.

${ }^{6}$ While seeds have the option of not complying (i.e., not posting reviews), they are very likely to comply because non-compliance can disqualify them from being a seed in the future.
} 
- $\quad$ Same-brand and same-category non-seed WOM by specialists and generalists.

Non-seed WOM that does not mention the focal product but mentions either the same brand (but products in different categories) or the same category (but competing brands) can occur as posts in either specialist or generalist forums.

\subsection{Data Collection}

Online WOM data. Our data comes from Naver's cosmetics forums and covers a 46month period (February 2008 to November 2011). ${ }^{7}$ The data were collected as follows. First, we obtained a list of all products that were focal products in SMCs in the three largest specialist cosmetics forums (Online Café). Second, we associated brand names and category names with each product. Third, we collected all focal product seed and non-seed WOM corresponding to these SMCs in the specialist forums. Fourth, we collected all focal product non-seed WOM corresponding to these SMCs in the generalist forums. Fifth, for the same time periods as the SMCs, we collected all posts in specialist and generalist communities that mentioned a focal product's brand or category (i.e., same-brand and same-category non-seed WOM by specialists and generalists). We also obtained data (for use as control variables) on some characteristics of the seeds' reviews (numbers of images, numbers of words), and whether the seed was designated as a "top reviewer" (indicated by a publicly observable icon beside their name; top reviewers are the top-10\% most active reviewers). Note that we excluded products that had seed WOM but no non-seed WOM, as well as products that were promoted with more than one SMC at the same time (since resultant non-seed WOM in that case cannot be attributed to a single SMC). ${ }^{8}$

\footnotetext{
${ }^{7}$ These forums are typically very large in terms of membership. For example, our data focuses on the largest three "Online Café" forums for cosmetics, and the average number of members is 153,761.

${ }^{8}$ Sixty-six products from 37 brands were excluded on this basis. These products came from a variety of brands and categories, distributed across categories similarly to the 390 included products.
} 
Our dataset consists of the SMC-related seed and non-seed WOM for 390 cosmetic products from 192 different brands across 11 separate cosmetics product categories (e.g., nail polish, toner, face mask, lipstick). For each of the 390 campaigns, our data is weekly and starts in the week in which the first focal product seed WOM is observed $(t=0)$ and continues until no more seed or non-seed WOM posts are observed for the focal product in any of the forums (or, at the latest, the end of the data-collection period). The mean number of weeks per campaign is $75.47(\mathrm{SD}=39.68, \min .=12, \max .=159)$.

Controls for other potential drivers of WOM activity. An alternative explanation for WOM-based effects is that non-seed WOM occurs not due to seed WOM but instead as a result of other actions taken by marketers that we do not observe. For example, traditional/offline advertising could affect online WOM. Also, more generally, the topicality or prominence of a given product could also affect the generation of WOM through a non-social mechanism. To control for these possibilities, we obtained data from other sources that tracked weekly advertising activity and topicality.

First, to control for advertising, we obtained weekly advertising spending data from Nielsen South Korea. For each campaign, they report the amount of money (in Korean Won) spent separately on television advertising and print advertising per week. ${ }^{9}$ They also provided weekly television and print advertising spending at the brand and category levels. Thus, we have six weekly advertising spending variables that we use to control for the possibility that advertising for the focal product, or at the brand or category levels, influences WOM activity. Note that in addition to advertising spending, Nielsen reports advertising volume data; i.e., the number of advertisements of each type placed per week. For product, brand, and category levels

\footnotetext{
${ }^{9}$ Only TV and print data were available. TV and print were the largest advertising media in Korea at the time of data collection (e.g., according to the Korean Broadcast Advertising Corporation, in 2011, combined TV and print spend was over $64 \%$ of total ad spending).
} 
of advertising, volume and spending variables were highly positively correlated. We use spending data in our analysis, and the results did not differ when volume data was used instead.

Second, topicality or cultural prominence might affect WOM about a product. While it is not possible to measure this directly, we use internet search activity as a proxy. The search activity for a given product should be positively correlated with how topical or culturally prominent it is at a given point in time. In other words, if something is topical, people should be more likely to search for it on the internet than something that is less topical (particularly in a country like South Korea that has extremely high levels of internet usage). We obtained weekly internet search trends data from $\mathrm{Naver}^{10}$ for all combinations of brand and category keywords (e.g., Chanel AND lipstick) corresponding to each of products in the SMCs in our dataset.

\subsection{Variables}

For each of the 390 products promoted through an SMC, we have weekly data capturing seed and non-seed WOM for the focal product, products from the same brand in different categories, and products from different brands in the same category. The main variables used in our analysis are defined in Table 1, and control variables are defined in Table 2.

\section{[INSERT TABLES $1 \& 2$ ABOUT HERE]}

Note that the indicator variable Utilitarian (Table 1), which we use as a moderating variable to test $\mathrm{H} 4$, was determined by looking at the category of product in each SMC and classifying it as either more utilitarian (Utilitarian $=1)$ or not $($ Utilitarian $=0)$. Even though cosmetics and beauty products could, in general, be thought of as quite hedonic given that they appeal to beauty ideals, in reality some of these products are perceived as more utilitarian

\footnotetext{
${ }^{10}$ Naver was more popular than Google in South Korea at the time of data collection. Also, Naver search "trends" data are similar Google Trends data for the U.S. and other countries.
} 
because they can deliver a variety of "functional" benefits to consumers. Many cosmetics products such as skincare creams or anti-aging ointments promise primarily functional benefits to consumers. Products that neither promise nor deliver particular functional benefits and are purely "cosmetic," such as many makeup products (e.g., lipstick), are more hedonic in nature. In our case, the products in our dataset that we determined to be of a more utilitarian nature were from the following categories: ${ }^{11}$ face masks, skin creams/essences/toners, hand/body creams, cleaners, functional products, hair treatments, tooth products, and male-oriented products. The other categories, in which the products were classified as of a more hedonic nature, were the following: face makeup, color makeup, lipstick, and perfume.

\section{Empirical Model}

To test our hypotheses, we are interested in how FocalSeedSpecialist ${ }_{j t}$, which tracks the number of posts about the focal product in SMC $j$ in week $t$ by seeds, affects each of six outcome variables that measure amount of WOM generated by non-seed consumers. Specifically, for testing H1 about focal product spillover effects, we estimate how FocalSeedSpecialist $t_{t}$ affects the amount of non-seed WOM generated about the focal product (FocalNonseedSpecialist ${ }_{j}$, FocalNonseedGeneralist ${ }_{j t}$ ), expecting positive effects. For testing $\mathrm{H} 2$ about brand spillover effects, we estimate how FocalSeedSpecialist ${ }_{j t}$ affects the amount of non-seed WOM generated about other products from the same brand as the focal product (BrandNonseedSpecialist $t_{t}$, BrandNonseedGeneralist $_{j t}$ ), expecting negative effects. For testing $\mathrm{H} 3$ about category spillover effects, we estimate how FocalSeedSpecialist ${ }_{j t}$ affects the amount of non-seed WOM generated about other products from different brands but in the same category as the focal product

\footnotetext{
${ }^{11}$ These product categories correspond to product classification from the Naver forums.
} 
(CategoryNonseedSpecialist ${ }_{j t}$, CategoryNonseedGeneralist $t_{j t}$ ), expecting negative effects. Finally, for testing H4, we expect Utilitarian ${ }_{j}$ to interact with FocalSeedSpecialist ${ }_{j t}$ such that the expected effects in $\mathrm{H} 2$ and $\mathrm{H} 3$ occur only when the focal product is of a more utilitarian nature $\left(\right.$ Utilitarian $\left._{j}=1\right)$.

Additionally, although not a focus of our conceptualization, we allow for a number of other variables to also be influenced by seeding activity and the other variables in this system. The following ten variables track seed WOM for the focal product, seed WOM for other products from the same brand as the focal product in campaign $j$, seed WOM for other products from the same category as the focal product in campaign $j$, and the advertising spending variables: FocalSeedSpecialist ${ }_{j t}$, BrandSeedSpecialist $_{j t}$, CategorySeedSpecialist $_{j t}$, SearchTrends $_{j t}$. FocalTVSpend $_{j t}$, FocalPrintSpend $_{j t}$, BrandTVSpend $_{j t}$, BrandPrintSpend $_{j t}$, CategoryTVSpend $_{j t}$, and CategoryPrintSpend ${ }_{j t}$.

Thus, in total we have 16 outcome variables that we treat as a multivariate dependent variable for modeling purposes (i.e., each is treated as an endogenous outcome). Thus, for testing our hypotheses we use a sixteen-equation multivariate model (one equation for each of the six endogenous outcome variables and one for each of the ten endogenous control variables) to estimate the hypothesized WOM spillover effects. Specifically, we log-transformed each variable (after adding one to avoid logarithms of zero) and estimated a multivariate dynamic model with campaign fixed effects to account for unobserved campaign-level heterogeneity. The multivariate specification (i.e., an endogenous system of equations) was used to allow for interdependencies between the sixteen outcome variables through correlated errors. Within each equation, each outcome was regressed on a one-week lag of itself, one-week lags of each of the other outcomes, and each of the exogenous control variables. For campaign $j$ and week $t$ the model is as follows: 
(1)

$$
\log \left(Y_{j t}+1\right)=A+\Lambda \log \left(Y_{j, t-1}+1\right)+\mathrm{B} X_{j t}+\Gamma M_{j, t-1}+u_{j}+e_{j t}
$$

$$
Y_{j t}=\left[\begin{array}{c}
\text { FocalSeedSpecialist }_{j t} \\
\text { FocalNonseedSpecialist }_{j t} \\
\text { FocalNonseedGeneralist }_{j t} \\
\text { BrandNonseedSpecialist }_{j t} \\
\text { BrandNonseedGeneralist }_{j t} \\
\text { CategoryNonseedSpecialist }_{j t} \\
\text { CategoryNonseedGeneralist }_{j t} \\
\text { BrandSeedSpecialist }_{j t} \\
\text { CategorySeedSpecialist }_{j t} \\
\text { SearchTrends }_{j t} \\
\text { FocalTVSpend }_{j t} \\
\text { FocalPrintSpend }_{j t} \\
\text { BrandTVSpend }_{j t} \\
\text { BrandPrintSpend }_{j t} \\
\text { CategoryTVSpend }_{j t} \\
\text { CategoryPrintSpend }_{j t}
\end{array}\right], X_{j t}=\left[\begin{array}{c}
\text { AvgWords }_{j t} \\
\text { AvgImages }_{j t} \\
\text { TopReviewer }_{j t} \\
\text { CategorySMC }_{j t} \\
\text { CategorySMC }_{j t} \\
\text { CategorySMC }_{j t}
\end{array}\right]
$$

$$
M_{j, t-1}=\left[\begin{array}{c}
\log \left(\text { FocalSeedSpecialist }_{j, t-1}+1\right) * \text { Utilitarian }_{j} \\
\text { Utilitarian }_{j}
\end{array}\right]
$$

Where: (1) A is an intercept vector, (2) $\Lambda$ is the matrix of effects of the lagged endogenous variables on the endogenous variables in $Y_{j t}$, (3) B is the matrix of effects of the exogenous control variables on the endogenous variables in $Y_{j t}$, (4) $\Gamma$ is the matrix of effects for the interaction $M_{j, t-1},(5) u_{j}$ are campaign fixed effects, and (6) $e_{j t} \sim \mathrm{N}(\mathbf{0}, \Sigma)$ where $\Sigma$ is a full unrestricted covariance matrix. $^{12}$

\section{Results}

\subsection{Descriptive Statistics}

${ }^{12}$ Our model is quite similar to other kinds of simultaneous/multi-equation models in marketing, e.g., VAR. 
Our results are based on 28,654 weekly observations for 390 SMCs for cosmetic products from 192 different brands and representing 11 categories. Descriptive statistics and correlations are reported in Tables 3-5. Per SMC, the average number of posts generated by seeds was 69.20 $($ median $=55, \mathrm{SD}=52.18, \min .=5, \max .=280)$. Generally, across campaigns, seeds' posting activities in any week, on average, however, were low $(\mathrm{M}=.94$, median $=0, \mathrm{SD}=7.321$, $\min .=$ 0, max. = 273). Not surprisingly, the first few weeks of a SMC typically produced the most WOM activity from seeds. For example, in the first four weeks of a campaign, the seeds posted an average of 49.67 posts $(\mathrm{SD}=38.43, \min .=0, \max .=281$, median $=40)$. Moreover, in the typical campaign, $16 \%$ of the total number of seeds' posts for a campaign occurred in the first two weeks, and $95 \%$ of the total seeds' posts were occurred within six weeks.

\section{[INSERT TABLES 3-5 ABOUT HERE]}

Non-seed WOM was generally more prevalent than seed WOM, as expected. Posts about the focal product by non-seeds in specialist forums (FocalNonseedSpecialist) ranged from zero to 2,340 posts $(\mathrm{M}=73.33, \mathrm{SD}=220.17$, median $=11)$, and from 0 to $2,272(\mathrm{M}=28.39, \mathrm{SD}=$ 165.04, median $=0$ ) in generalist forums (FocalNonseedGeneralist). Also, non-seed WOM posts were generated at a slower rate than seeds' posts. For example, for the average campaign, only $7 \%$ of the total number of non-seed WOM posts (across specialist and generalist forums) occurred in the first two weeks of the campaign (vs. $16 \%$ for seed WOM), and only $26 \%$ occurred by the end of the sixth week (vs. 95\% for seed WOM). This is consistent with our general prediction that WOM by seeds triggers non-seed WOM activity.

\subsection{Focal Product, Brand, and Category Spillover Effects}

We begin by testing H1, H2, and H3. For this, we estimated the model described above but excluding the moderating effect of $\operatorname{Utilitarian}_{j}$ (i.e., $\Gamma=0$ in equation 1 ). The fit statistics 
were $-2 \mathrm{LL}=521,674, \mathrm{AIC}=522,682$, and $\mathrm{BIC}=526,846$. Two alternative models were estimated, using different combinations of control variables. One model did not include controls for other SMC activities in the category, i.e., no CategorySMC1, CategorySMC2, or CategorySMC3 effects $(-2 \mathrm{LL}=560,998 ; \mathrm{AIC}=561,911 ; \mathrm{BIC}=565,691)$. Another model had additional controls measuring the amounts of non-seed WOM for focal product, brand, and category broken down into specialist and generalist sources that had occurred prior to each SMC $(-2 \mathrm{LL}=521,634 ; \mathrm{AIC}=522,834 ; \mathrm{BIC}=527,792)$. The main model fit better than either alternative, and thus the results reported next are based on it.

Parameter estimates for the full model of spillover effects are reported in Tables 6 (focal product spillovers), 7 (brand spillovers), and 8 (category spillovers). These tables cover the six outcome variables. Parameter estimates for other endogenous variables are reported in the Web Appendix (Tables WA1-6), as is the error correlation matrix (Table WA7).

\section{[INSERT TABLES 6-8 ABOUT HERE]}

For the focal product spillover effects (Table 6), the effect of seed WOM on non-seed focal product WOM among specialists was positive and significant $(.041, \mathrm{SE}=.004, \mathrm{p}<.001)$. The effect of seed WOM on non-seed focal product WOM among generalists was also positive and significant $(.005, \mathrm{SE}=.003, \mathrm{p}=.046)$. These results indicate positive focal product spillover effects and thus support $\mathrm{H} 1$. We also find significant indirect paths whereby non-seed focal product WOM among specialists also positively affects non-seed focal product WOM among generalists $(.015, \mathrm{SE}=.003, \mathrm{p}<.001)$ and vice versa $(.050, \mathrm{SE}=.007, \mathrm{p}<.001)$.

For the brand spillover effects (Table 7), the effect of seed WOM on non-seed WOM about other products from the same brand as the focal product was negative and significant for generalists $(-.007, \mathrm{SE}=.003, \mathrm{p}=.012)$, and negative but not significant for specialists $(-.004, \mathrm{SE}$ $=.004, \mathrm{p}=.366)$. There was a positive and significant effect of BrandNonseedGeneralist on 
BrandNonseedSpecialist $(.040, \mathrm{SE}=.006, \mathrm{p}<.001)$, however, which means that seed WOM indirectly triggered a negative brand spillover effect for specialists even though the direct effect was not significant. These results are consistent with $\mathrm{H} 2$.

For the category spillover effects (Table 8), the effect of seed WOM on non-seed WOM about other products from different brands was negative and significant for specialists (-.012, SE $=.004, \mathrm{p}=.003)$, and negative but not significant for generalists $(-.010, \mathrm{SE}=.006, \mathrm{p}=.138)$. There was a positive and significant effect of CategoryNonseedSpecialist on CategoryNonseedGeneralist $(.137, \mathrm{SE}=.008, \mathrm{p}<.001)$, however, which means that seed WOM indirectly triggered a negative category spillover effect for generalists even though the direct effect was not significant. These results are consistent with $\mathrm{H} 3$.

\subsection{Process Evidence: Moderation by Utilitarian}

Finally, we test H4, which hypothesizes that the negative brand and category spillover effects reported above occur when an SMC's focal product is more utilitarian in nature. To test this we added the effect of Utilitarian and its interaction with FocalSeedSpecialist to the model (i.e., $\Gamma$ in equation 1 was not fixed to 0 ).

The results supported H4(a) and H4(b). For brand spillovers, as reported above, the direct negative brand spillover effect was found for generalists but not for specialists, so we focus on BrandNonseedGeneralist here. The interaction between FocalSeedSpecialist and Utilitarian had a negative and significant effect on BrandNonseedGeneralist (interaction $=-.011, \mathrm{SE}=.004, \mathrm{p}$ $=.014)$. In terms of simple effects, this brand spillover effect was negative and significant only when the product was more utilitarian in nature $($ simple effect $=-.011, \mathrm{SE}=.003, \mathrm{p}=.001) .{ }^{13}$ For category spillovers, as reported above, the direct negative category spillover effect was

${ }^{13}$ The corresponding simple effect for when Utilitarian $=0$ was $.000, \mathrm{SE}=.004, \mathrm{p}=.978$. 
found for specialists but not for generalists, so we focus on CategoryNonseedSpecialist here. The interaction between FocalSeedSpecialist and Utilitarian had a negative and significant effect on CategoryNonseedSpecialist (interaction $=-.023, \mathrm{SE}=.008, \mathrm{p}=.006)$. In terms of simple effects, this category spillover effect was negative and significant only when the product was more utilitarian in nature $($ simple effect $=-.020, \mathrm{SE}=.004, \mathrm{p}<.001) .{ }^{14}$

\section{Discussion and Conclusion}

Seeding and related approaches such as influencer marketing and social referral programs have become increasingly popular among marketers and continue to be the focus of a growing stream of literature. However, while prior research focuses mainly on aspects related to SMC design, optimal seed selection, and whether SMCs produce positive results with respect to WOM generation and sales of a focal or featured product, the literature has not considered some of the broader consequences of firm-initiated seeded marketing programs. The goal of the current research was to take a first step in this direction by introducing and empirically testing a set of brand- and category-related WOM spillover effects in the context of SMCs carried out in online settings. Generally, our findings indicate that SMC-triggered WOM by seed consumers can spur a variety of spillover effects at the focal product, brand, and category levels. Moreover, these spillover effects vary in their size, with focal product spillovers being the largest and brand spillovers being the smallest. Consistent with our conceptualization, we found that SMCs generate more non-seed WOM about a focal product, and can also reduce WOM about related products at the brand and category levels.

\footnotetext{
${ }^{14}$ The corresponding simple effect for when Utilitarian $=0$ was $.003, \mathrm{SE}=.008, \mathrm{p}=.667$.
} 
The negative brand and category spillover effects are counterintuitive given that much of the prior literature on spillovers in other marketing contexts finds positive effects. The fact that we find negative, instead of positive, brand and category spillover effects in the SMC context underscores the more general point that SMCs - which rely on WOM generated by seed consumers - are different from other, more traditional forms of marketing communication. More specifically, our findings suggest that seeding focal products with specialist consumers and encouraging them to generate WOM can serve an additional purpose for marketers in terms of helping non-seed consumers focus on the focal product when generating their own WOM. In other words, in line with our theory and empirical evidence, SMCs can make consumers—seeds and non-seeds - more inclined to construe focal products more concretely and thus suppress broader thoughts that might have otherwise led to brand- and/or category-level WOM.

While traditional advertising literature typically considers positive brand spillovers as beneficial for the firm (e.g., when advertising one product also lifts awareness for other products under the same brand), the negative WOM brand spillover effects that we identified might not necessarily spell bad news for marketers. Firms may value the ability of SMCs to help non-seed consumers concentrate on the focal product and reduce buzz about other products under the same brand in a variety of market settings. For example, this could help a firm when it launches a new product and wants attention to be focused on that product and not other products in the brand portfolio. It may also be helpful when there is a substantial variation in product quality or appeal across products under the same brand and a firm does not want negative brand associations to contaminate WOM and consumers' perceptions about a focal product in an SMC. Finally, marketers may simply not want to have to "compete" against themselves for WOM when running an SMC for a particular product in their lineup, and the negative brand spillover effects suggest that this is unlikely to be the case (particularly for generalist consumers). 
The presence of negative category spillovers is important from a competitive standpoint. It suggests that firms could benefit from SMCs not only through the positive effect on WOM for the own focal product, but also through the negative effect on WOM for competitors' products in the same category as the focal product. Taken a step further, this implies that firms could strategically deploy SMCs to mitigate WOM for competitors' products in the same category. ${ }^{15}$ Similar to the case of brand spillovers, negative category spillover effects may also make firms using SMCs better or worse off depending on the market environment. For example, when capturing higher market share is important for a firm's success, a negative effect on competitors' WOM is beneficial; however, firms with dominant market positions that are primarily interested in growing the overall market size would not find such an effect to be a beneficial externality of running an SMC.

We also identified the importance of product type for the relative strengths of both brand and category negative spillover effects. This helps firms to determine for which products SMCs can be deployed to take advantage of the spillover effects in various contexts we outlined above. For example, companies looking to reduce WOM for competitors' brands should rely on SMCs for products that are more utilitarian, rather than more hedonic. Firms can also influence the spillover effects by suggesting that seed consumers use more specific terms for describing the focal product if they want to benefit from negative brand and/or category spillover effects. Or, conversely, if they wish to prevent such effects, they can promote the use of more general, abstract WOM generated by seeds.

The current study is not without limitations. First, our data does not account for WOM valence (only volume). This is because reliable sentiment analysis algorithms are not available

\footnotetext{
${ }^{15}$ This is in contrast with previous findings on positive category spillovers in other domains of marketing communications, such as advertising (e.g., Lewis and Nguyen 2015; Sahni 2013).
} 
for the Korean language, and manually scoring post sentiment is infeasible given the large number of posts. However, this is only worrisome if there is substantial variation in valence across forum posts and campaigns. To assess the likelihood of this, we randomly sampled 30 campaigns (out of 390) and had a native Korean speaker manually read the collected posts and judge their valence. The native speaker found most of the posts were positive: opinions judged as "mostly negative" accounted for a mere $1.3 \%$ of total WOM volume in the sample (which covered both specialist and generalist forums), and opinions judged as "mixed" were $4.3 \%$ and $1.3 \%$ of WOM volume in the specialist and generalist forums, respectively. Thus, our findings are based on predominantly positive seed and non-seed WOM (similar to the online community data in Stephen and Galak 2012). Nevertheless, it would be interesting for future research to test our WOM spillovers typology in contexts where WOM valence exhibits greater variance. Second, the current study is limited to the cosmetics industry. Although it is large and features many distinct categories, it would be interesting to examine different industries and learn how the identified spillover effects might vary. Such an analysis could possibly extend recent work on different types of products in viral/WOM marketing contexts (e.g., Lovett, Peres, and Shachar 2013; Schulze, Schöler, and Skiera 2014). Finally, due to the nature of our data, we could not consider consumer- or network-related factors beyond specialist or generalist membership.

In conclusion, this research addresses an important aspect of online WOM campaigns that has received scant attention in extant literature: the broader consequences of SMCs in terms of spillover effects. Our findings not only uncover the presence of WOM spillover effects as consequences of SMCs but also underscore the importance of taking these into account when planning SMCs and assessing the value of such programs. We hope this study spurs additional research on this and related topics. 


\section{References}

Anderson, Eugene W. (2003a), "The Formation of Market-Level Expectations and Its Covariates," Journal of Consumer Research, 30(1), 115-124.

Anderson, Michael C. (2003b), " Rethinking Interference Theory: Executive Control and the Mechanisms of Forgetting," Journal of Memory and Language, 49(4), 415-445.

Anderson, Michael C. and Theodore Bell (2001), "Forgetting Our Facts: The Role of Inhibitory Processes in the Loss of Propositional Knowledge," Journal of Experimental Psychology, General, 130, 544-570.

Anderson, Michael C. and Robert A. Bjork (1994), "Mechanisms of Inhibition in Long-Term Memory: A New Taxonomy,” in D. Dagenbach, \& T. Carr (Eds.), Inhibitory processes in attention, memory and language (pp. 265-326). San Diego: Academic Press.

Anderson Eric T. and Duncan Simester (2013), “Advertising in a Competitive Market: The Role of Product Standards, Customer Learning, and Switching Costs," Journal of Marketing Research, 50(4), 489-504.

Arndt, Johan (1967), "Role of Product-Related Conversations in the Diffusion of A New Product," Journal of Marketing Research, 4(3), 291-295.

Balachander, Subramanian, and Sanjoy Ghose (2003), "Reciprocal Spillover Effects: a Strategic Benefit of Brand Extensions," Journal of Marketing, 67(1), 4-13.

Berger, Jonah and Eric M. Schwartz (2011), "What Drives Immediate and Ongoing Word of Mouth?” Journal of Marketing Research, 48(5), 869-880.

Biyalogorsky, Eyal, Eitan Gerstner, and Barak Libai (2001), “Customer Referral Management: Optimal Reward Program,” Marketing Science, 20(1), 82-95.

Bone, Paula F. (1995), “Word-of-mouth effects on short-term and long-term product judgments", Journal of Business Research, 32(3), 213-223.

Borah, Abhishek, and Gerard J. Tellis (2015), "Halo (Spillover) Effects in Social Media: Do Product Recalls of One Brand Hurt or Help Rival Brands?" Journal of Marketing Research, in press.

Chevalier, Judith A. and Dina Mayzlin (2006), "The Effect of Word or Mouth on Sales: Online Book Reviews," Journal of Marketing Research, 43(3), 345-354. 
Dhar, Ravi and Eunice Y. Kim (2007), "Seeing the Forest or the Trees: Implications of Construal Level Theory for Consumer Choice," Journal of Consumer Psychology, 17(2), 96-100.

Erdem, Tülin and Baohong Sun (2002), "An Empirical Investigation of the Spillover Effects of Advertising and Sales Promotions in Umbrella Branding," Journal of Marketing Research, 39(4), 408-420.

Euromonitor (2013), Color Cosmetics in South Korea, Euromonitor International.

Fiedler, Klaus (2007), "Construal Level Theory as an Integrative Framework for Behavioral Decision-Making Research and Consumer Psychology," Journal of Consumer Psychology, 17(2), 101-106.

Garthwaite, Craig L. (2014), "Demand Spillovers, Combative Advertising, and Celebrity Endorsements," American Economic Journal: Applied Economics, 6(2), 76-104.

Godes, David and Dina Mayzlin (2004), "Using Online Conversations to Study Word-Of-Mouth Communication," Marketing Science, 23(4), 545-515.

Godes, David and Dina Mayzlin (2009), "Firm-Created Word-of-Mouth Communication:

Evidence from a Field Test," Marketing Science, 28(4), 721-739.

Herr, Paul M., Frank R. Kardes, and John Kim (1991), "Effects of Word-Of-Mouth and ProductAttribute Information on Persuasion: An Accessibility-Diagnosticity Perspective," Journal of Consumer Research, 17(4), 454-462.

Hinz, Oliver, Bernd Skiera, Christian Barrot, and Jan U. Becker (2011), "Seeding Strategy for Viral Marketing: An Empirical Comparison,” Journal of Marketing, 75(6), 55-71.

Kardes, Frank R., Maria L. Cronley, and John Kim (2006), "Construal-Level Effects on Preference Stability, Preference-Behavior Correspondence, and the Suppression of Competing Brands," Journal of Consumer Psychology, 16(2), 135-144.

Katz, Elihu and Paul F. Lazarsfeld (1955), Personal Influence, New York: The Free Press. Kornish, Laura J., and Qiuping Li (2010), "Optimal Referral Bonuses with Asymmetric Information: Firm-Offered And Interpersonal Incentives," Marketing Science, 29(1), 108-121. Kozinets, Robert V., Kristine De Valck, Andrea C. Wojnicki, and Sarah JS Wilner (2010), "Networked Narratives: Understanding Word-of-Mouth Marketing in Online Communities," Journal of Marketing, 74(2), 71-89. 
Krishnan, Trichy, P.B. Seetharaman and Demetrios Vakratsas (2012), “The Multiple Roles of Interpersonal Communication in New Product Growth," International Journal of Research in Marketing, 29(3), 292-305.

Kumar, V., J. Andrew Petersen, and Robert P. Leone (2010), “Driving Profitability by Encouraging Customer Referrals: Who, When, and How," Journal of Marketing, 74(5), 1-17. Lamberton, Cait and Andrew T. Stephen (2015), "Taking Stock of the Digital Revolution: A Critical Analysis and Agenda for Digital, Social Media, and Mobile Marketing Research,” working paper, Saïd Business School, Oxford.

Lewis, Randall A., and Dan Nguyen (2015), "Display Advertising's Competitive Spillovers to Consumer Search," Quantitative Marketing and Economics, 13(2), 93-115.

Libai, Barak, Ruth Bolton, Marnix S. Bügel, Ko de Ruyter, Oliver Götz, Hans Risselada, and Andrew T. Stephen (2010), “Customer-to-Customer Interactions: Broadening the Scope of Word-of-Mouth Research,” Journal of Service Research, 13(3), 267-282.

Libai, Barak, Eitan Muller, and Renana Peres (2009), "The Role of Within-Brand and CrossBrand Communications in Competitive Growth," Journal of Marketing, 73(3), 19-34.

Libai, Barak, Eitan Muller and Renana Peres (2013), "Decomposing the Value of Word-ofMouth Seeding Programs: Acceleration Versus Expansion," Journal of Marketing Research, 50(2), 161-176.

Liberman, Nira and Yaacov Trope (1998), "The Role of Feasibility and Desirability Considerations in Near and Distant Future Decisions: a Test of Temporal Construal Theory," Journal of Personality and Social Psychology, 75(1), 5.

Lovett, Mitchell J., Renana Peres, and Ron Shachar (2013), "On Brands and Word of Mouth," Journal of Marketing Research, 50(2), 427-444.

Moore, Sarah G (2015), "Attitude Predictability and Helpfulness in Online Reviews: The Role of Explained Actions and Reactions," Journal of Consumer Research, 42(1), 30-44.

Peres, Renana and Christophe Van den Bulte (2014), "When to Take or Forgo New Product Exclusivity: Balancing Protection from Competition Against Word-of-Mouth Spillover," Journal of Marketing, 78(2), 83-100.

Rogers Everett, M. (1995), Diffusion of Innovations. New York. Ryu, Gangseog and Lawrence Feick (2007), “A Penny for Your Thoughts: Referral Reward Programs and Referral Likelihood,” Journal of Marketing, 71(1), 84-94. 
Sahni, Navdeep (2013), “Advertising Spillovers: Field-Experiment Evidence and Implications for Returns from Advertising," Working paper.

Schulze, Christian, Lisa Schöler, and Bernd Skiera (2014), "Not All Fun and Games: Viral Marketing for Utilitarian Products," Journal of Marketing, 78(1), 1-19.

Schmitt, Philipp, Bernd Skiera, and Christophe Van den Bulte (2011), "Referral Programs and Customer Value," Journal of Marketing, 75(1), 46-59.

Simonin, Bernard L. and Julie A. Ruth (1998), "Is a Company Known by the Company it Keeps? Assessing the Spillover Effects of Brand Alliances on Consumer Brand Attitudes," Journal of Marketing Research, 30-42.

Sonnier, Garrett P., Leigh McAlister, and Oliver J. Rutz (2011), “A Dynamic Model of the Effect of Online Communications on Firm Sales," Marketing Science, 30(4), 702-716.

Stephen, Andrew T. (2016), "The Role of Digital and Social Media Marketing In Consumer Behavior," Current Opinion in Psychology, 10 (August), 17-21.

Stephen, Andrew T. and Jeff Galak (2012), "The Effects of Traditional and Social Earned Media on Sales: A Study of a Microlending Marketplace," Journal of Marketing Research, 49(5), 629-639.

Stephen, Andrew T. and Donald Lehmann (2015), "Designing Incentives For Word-Of-Mouth Communications That Speed Up Information Diffusion: How Positive Externalities Affect Customers' Transmission Behaviors," Working paper.

The Economist (2014), "Now or Naver," March 1.

Trope, Yaacov and Nira Liberman (2003), "Temporal Construal," Psychological Review, 110(3), 403-421.

Trope, Yaacov and Nira Liberman (2010), "Construal-Level Theory of Psychological Distance," Psychological Review, 117(2), 440-463.

Trope, Yaacov, Nira Liberman, and Cheryl Wakslak (2007), "Construal Levels and Psychological Distance: Effects on Representation, Prediction, Evaluation, and Behavior," Journal of Consumer Psychology, 17(2), 83-95.

Trusov, Michael, Randolph E. Bucklin, and Koen Pauwels (2009), "Effects of Word-of-Mouth Versus Traditional Marketing: Findings from an Internet Social Networking Site," Journal of Marketing, 73(5), 90-102. 
Van den Bulte, Christophe and Yogesh V. Joshi (2007), "New Product Diffusion with Influentials and Imitators," Marketing Science, 26(3), 400-421.

Van den Bulte, Christophe and Stefan Wuyts (2007), Social Networks and Marketing. Cambridge, MA: Marketing Science Institute.

Villanueva, Julian, Shijin Yoo, and Dominique M. Hanssens (2008), “The Impact of MarketingInduced Versus Word-of-Mouth Customer Acquisition on Customer Equity Growth," Journal of Marketing Research, 45(1), 48-59.

Watts, Duncan J. and Peter S. Dodds (2007), “Influentials, Networks, and Public Opinion Formation," Journal of Consumer Research, 34(4), 441-458.

Whyte, William H. Jr. (1954), "The Web of Word Of Mouth," Fortune, 50 (1954), 140-143.

Wojnicki, Andrea C., and David Godes (2008), "Word-Of-Mouth as Self-Enhancement," HBS Marketing Research Paper 06-01.

Word of Mouth Marketing Association (2005), "Word of Mouth 101”, Chicago, IL.

Word of Mouth Marketing Association (2014), "The State of Word of Mouth Marketing: A Survey of Marketers", Chicago, IL. 


\section{Table 1}

Main Variables Used in Analysis

\begin{tabular}{ll}
\hline Variable & Definition \\
\hline Antecedent: & $\begin{array}{l}\text { The number of posts about the focal product in campaign } j \text { in week } t \text { by } \\
\text { seeds in specialist forums; i.e., focal product seed WOM. }\end{array}$ \\
\hline FocalSeedSpecialist $\mathrm{j}_{\mathrm{jt}}$ & $\begin{array}{l}\text { Equal to } 1 \text { if the focal product in campaign } j \text { is more utilitarian in nature. } \\
\text { Otherwise equal to } 0 .\end{array}$ \\
\hline Utilitarian $_{\mathrm{j}}$ & $\begin{array}{l}\text { Ooderator (for testing H4): } \\
\end{array}$
\end{tabular}

\section{Outcomes:}

FocalNonseedSpecialist ${ }_{\mathrm{jt}}$

FocalNonseedGeneralist $_{\mathrm{jt}}$

The number of posts about the focal product in campaign $j$ in week $t$ by non-seeds in specialist forums; i.e., focal product non-seed WOM by specialists.

BrandNonseedSpecialist ${ }_{\mathrm{jt}}$

The number of posts about the focal product in campaign $j$ in week $t$ by non-seeds in generalist forums; i.e., focal product non-seed WOM by generalists.

The number of posts about the same-brand, different-category products related to campaign $j$ mentioned in week $t$ in specialist forums; i.e., samebrand non-seed WOM by specialists.

BrandNonseedGeneralist ${ }_{\mathrm{jt}} \quad$ The number of posts about the same-brand, different-category products related to campaign $j$ mentioned in week $t$ in generalist forums; i.e., samebrand non-seed WOM by generalists.

CategoryNonseedSpecialist ${ }_{\mathrm{jt}} \quad$ The number of posts about the different-brand, same-category products related to campaign $j$ mentioned in week $t$ in specialist forums; i.e., samecategory non-seed WOM by specialists.

CategoryNonseedGeneralist ${ }_{\mathrm{jt}}$ The number of posts about the different-brand, same-category products related to campaign $j$ mentioned in week $t$ in generalist forums; i.e., samecategory non-seed WOM by generalists. 
Table 2

Control Variables Used in Analysis

\begin{tabular}{ll}
\hline Variable & Definition \\
\hline $\begin{array}{l}\text { Other Seed WOM Activity: } \\
\text { BrandSeedSpecialist } t_{t}\end{array}$ & $\begin{array}{l}\text { The number of same-brand products related to campaign } j \text { mentioned in } \\
\text { week } t \text { in specialist forums by seeds; i.e., SMC activity for other products } \\
\text { from the same brand. } \\
\text { The number of same-category products related to campaign } j \text { mentioned in } \\
\text { week } t \text { in specialist forums by seeds; i.e., SMC activity for other products } \\
\text { in the same category. }\end{array}$ \\
\hline
\end{tabular}

Advertising Spending: $_{\text {FocalTVSpend }_{j t}}$
FocalPrintSpend $_{j t}$
BrandTVSpend $_{j t}$
BrandPrintSpend $_{j t}$
CategoryTVSpend $_{j t}$
CategoryPrintSpend $_{j t}$

Amount of money spent on television advertising for the product featured in campaign $j$ in week $t$.

Amount of money spent on print advertising for the product featured in campaign $j$ in week $t$.

Amount of money spent on television advertising for the brand featured in campaign $j$ in week $t$ (excluding for the focal product).

Amount of money spent on print advertising for the brand featured in campaign $j$ in week $t$ (excluding for the focal product).

Amount of money spent on television advertising for the category featured in campaign $j$ in week $t$ (excluding for the focal product).

Amount of money spent on print advertising for the category featured in campaign $j$ in week $t$ (excluding for the focal product).

Prominence/General Interest:
SearchTrends $s_{j t}$

Relative size of search trend volume (based on Naver) for combination of brand and category, corresponding to product being seeded in campaign $j$ in week $t$.

\footnotetext{
Review and Seed Characteristics:

Avg Words ${ }_{j t}$

The average number of words per seed WOM post for campaign $j$ in week $t$.

AvgImages $_{j t}$

The average number of images per seed WOM post for campaign $j$ in

TopReviewer $_{j t}$ week $t$.

The number of seed WOM posts for campaign $j$ in week $t$ posted by seeds that are designated as "top" reviewers.
}

\footnotetext{
Competing SMCs in the Category:

CategorySMC1 $_{j t} \quad$ Equal to 1 if there were other SMCs running in the category one week before week $t$. Otherwise equal to 0 .

CategorySMC2 $2_{j t} \quad$ Equal to 1 if there were other SMCs running in the category two weeks before week $t$. Otherwise equal to 0 .

CategorySMC3 $3_{j t} \quad$ Equal to 1 if there were other SMCs running in the category three weeks before week $t$. Otherwise equal to 0 .
} 
Table 3

Descriptive Statistics Per Campaign

\begin{tabular}{|c|c|c|c|c|c|}
\hline & Mean & Median & St. Dev. & Min. & Max. \\
\hline \multicolumn{6}{|l|}{ Endogenous Variables: } \\
\hline FocalNonseedSpecialist ${ }_{j t}$ & 73.33 & 11.0 & 220.17 & 0 & 2340 \\
\hline FocalNonseedGeneralist $_{j t}$ & 28.39 & 0 & 165.04 & 0 & 2272 \\
\hline BrandNonseedSpecialist $_{\mathrm{jt}}$ & 54.44 & 16.5 & 86.69 & 0 & 490 \\
\hline BrandNonseedGeneralist $\mathrm{jt}_{\mathrm{t}}$ & 17.88 & 0 & 45.19 & 0 & 294 \\
\hline CategoryNonseedSpecialist ${ }_{\mathrm{jt}}$ & 1759.68 & 1391.0 & 1419.32 & 0 & 10458 \\
\hline CategoryNonseedGeneralist $_{\mathrm{jt}}$ & 564.79 & 384.0 & 479.60 & 0 & 3406 \\
\hline FocalSeedSpecialist $t_{\mathrm{jt}}$ & 69.20 & 55.0 & 52.18 & 5 & 280 \\
\hline BrandSeedSpecialist $_{j t}$ & 23.27 & 5.0 & 41.23 & 0 & 227 \\
\hline CategorySeedSpecialist ${ }_{j t}$ & 405.17 & 330.0 & 336.75 & 0 & 1679 \\
\hline SearchTrends $_{\mathrm{jt}}$ & 6.40 & 0 & 22.92 & 0 & 182.64 \\
\hline FocalTVSpend $_{\mathrm{jt}}$ & 99234.23 & 0 & 1529553.26 & 0 & 30022941 \\
\hline FocalPrintSpend $_{\mathrm{jt}}$ & 31209.41 & 0 & 252985.44 & 0 & 4358316 \\
\hline BrandTVSpend $_{j t}$ & 546981.74 & 0 & 3891735.71 & 0 & 63364679 \\
\hline BrandPrintSpend $_{\mathrm{jt}}$ & 437786.46 & 0 & 1604884.78 & 0 & 19158026 \\
\hline CategoryTVSpend $_{\mathrm{jt}}$ & 77865207.44 & 52384018 & 74759803.14 & 0 & 261007283 \\
\hline CategoryPrintSpend $_{\mathrm{jt}}$ & 51106308.42 & 26484131 & 57328116.22 & 0 & 205134403 \\
\hline \multicolumn{6}{|l|}{ Control Variables: } \\
\hline AvgWords $_{\mathrm{jt}}$ & 13.29 & 8.88 & 12.82 & 1.29 & 73.75 \\
\hline AvgImages ${ }_{j t}$ & .72 & .45 & .73 & .01 & 4.87 \\
\hline TopReviewer $_{j t}$ & 6.25 & 4.0 & 16.59 & 0 & 189 \\
\hline
\end{tabular}


Table 4

Correlations Between Endogenous Variables

\begin{tabular}{|c|c|c|c|c|c|c|c|c|c|c|c|c|c|c|c|}
\hline & 1 & 2 & 3 & 4 & 5 & 6 & 7 & 8 & 9 & 10 & 11 & 12 & 13 & 14 & 15 \\
\hline 1. FocalNonseedSpecialist & & & & & & & & & & & & & & & \\
\hline 2. FocalNonseedGeneralist & .323 & & & & & & & & & & & & & & \\
\hline 3. BrandNonseedSpecialist & .105 & -.014 & & & & & & & & & & & & & \\
\hline 4. BrandNonseedGeneralist & .070 & .033 & .376 & & & & & & & & & & & & \\
\hline 5. CategoryNonseedSpecialist & .011 & -.013 & .125 & .013 & & & & & & & & & & & \\
\hline 6. CategoryNonseedGeneralist & .008 & -.005 & .086 & .045 & .675 & & & & & & & & & & \\
\hline 7. FocalSeedSpecialist & .107 & .043 & .001 & .002 & -.013 & -.005 & & & & & & & & & \\
\hline 8. BrandSeedSpecialist & -.093 & -.045 & .098 & .054 & .082 & .007 & -.027 & & & & & & & & \\
\hline 9. CategorySeedSpecialist & -.097 & -.035 & -.026 & -.070 & .485 & .217 & -.028 & .199 & & & & & & & \\
\hline 10. SearchTrends & .264 & .091 & .147 & .172 & .058 & .055 & .020 & -.089 & -.146 & & & & & & \\
\hline 11. FocalTVSpend & .051 & .006 & .022 & .037 & .018 & -.008 & .013 & -.025 & .063 & .201 & & & & & \\
\hline 12. FocalPrintSpend & .065 & .007 & .007 & .017 & .006 & -.004 & .007 & -.019 & .019 & .082 & .272 & & & & \\
\hline 13. BrandTVSpend & .097 & .029 & .036 & .073 & .039 & .035 & -.007 & -.057 & -.001 & .255 & .221 & .078 & & & \\
\hline 14. BrandPrintSpend & .104 & .015 & .067 & .045 & .025 & .008 & .002 & -.059 & -.009 & .163 & .151 & .194 & .339 & & \\
\hline 15. CategoryTVSpend & -.093 & -.005 & -.054 & -.010 & .119 & .127 & -.010 & .108 & .454 & -.133 & .025 & .009 & .003 & -.023 & \\
\hline 16. CategoryPrintSpend & -.050 & -.012 & -.014 & -.025 & .085 & -.038 & -.005 & .075 & .251 & -.063 & .030 & .071 & -.003 & .117 & .300 \\
\hline
\end{tabular}


Table 5

VIFs and Correlations Between One-Week Lagged and Current-Week Endogenous Variables

\begin{tabular}{|c|c|c|c|c|c|c|c|c|c|c|c|c|c|c|c|c|c|}
\hline & VIF & $\begin{array}{c}\text { Lag } \\
1\end{array}$ & $\begin{array}{c}\text { Lag } \\
2\end{array}$ & $\begin{array}{c}\text { Lag } \\
\mathbf{3}\end{array}$ & $\begin{array}{c}\text { Lag } \\
4\end{array}$ & $\begin{array}{c}\text { Lag } \\
5\end{array}$ & $\begin{array}{c}\text { Lag } \\
6 \\
\end{array}$ & $\begin{array}{c}\text { Lag } \\
7\end{array}$ & $\begin{array}{c}\text { Lag } \\
8\end{array}$ & $\begin{array}{c}\text { Lag } \\
9\end{array}$ & $\begin{array}{c}\text { Lag } \\
10\end{array}$ & $\begin{array}{c}\text { Lag } \\
11\end{array}$ & $\begin{array}{c}\text { Lag } \\
12 \\
\end{array}$ & $\begin{array}{c}\text { Lag } \\
13 \\
\end{array}$ & $\begin{array}{c}\text { Lag } \\
14 \\
\end{array}$ & $\begin{array}{c}\text { Lag } \\
15 \\
\end{array}$ & $\begin{array}{c}\text { Lag } \\
16\end{array}$ \\
\hline 1. FocalNonseedSpecialist & 1.23 & .776 & .318 & .095 & .067 & .005 & .006 & .100 & -.094 & -.097 & .257 & .050 & .074 & .097 & .113 & -.091 & -.049 \\
\hline 2. FocalNonseedGeneralist & 1.12 & .317 & .914 & -.013 & .030 & -.013 & -.008 & .045 & -.044 & -.036 & .088 & .006 & .010 & .028 & .012 & -.008 & -.014 \\
\hline 3. BrandNonseedSpecialist & 1.22 & .093 & -.014 & .723 & .356 & .106 & .075 & .003 & .094 & -.027 & .146 & .023 & .009 & .036 & .075 & -.050 & -.021 \\
\hline 4. BrandNonseedGeneralist & 1.20 & .068 & .028 & .370 & .679 & .012 & .022 & -.001 & .042 & -.070 & .174 & .037 & .010 & .072 & .039 & -.008 & -.020 \\
\hline 5. CategoryNonseedSpecialist & 2.56 & .010 & -.011 & .106 & .012 & .876 & .650 & -.012 & .078 & .475 & .058 & .018 & .008 & .038 & .022 & .118 & .061 \\
\hline 6. CategoryNonseedGeneralist & 2.00 & .008 & -.007 & .081 & .024 & .658 & .745 & -.007 & .005 & .211 & .055 & -.008 & -.006 & .035 & .008 & .127 & -.066 \\
\hline 7. FocalSeedSpecialist & 1.01 & .097 & .033 & .007 & .002 & -.009 & -.011 & .179 & -.027 & -.029 & .020 & .014 & .014 & -.006 & .000 & -.009 & -.005 \\
\hline 8. BrandSeedSpecialist & 1.07 & -.091 & -.044 & .099 & .056 & .083 & .007 & -.023 & .946 & .196 & -.089 & -.024 & -.018 & -.056 & -.056 & .104 & .072 \\
\hline 9. CategorySeedSpecialist & 1.86 & -.095 & -.033 & -.025 & -.069 & .473 & .212 & -.028 & .196 & .970 & -.145 & .063 & .019 & -.003 & -.011 & .447 & .231 \\
\hline 10. SearchTrends & 1.25 & .258 & .090 & .147 & .172 & .058 & .057 & .018 & -.089 & -.147 & .938 & .201 & .090 & .256 & .166 & -.133 & -.062 \\
\hline 11. FocalTVSpend & 1.16 & .050 & .005 & .023 & .036 & .019 & -.008 & .014 & -.025 & .063 & .200 & .923 & .275 & .202 & .110 & .022 & .025 \\
\hline 12. FocalPrintSpend & 1.11 & .068 & .010 & .009 & .012 & .008 & -.001 & .017 & -.019 & .022 & .078 & .241 & .106 & .081 & .033 & .011 & -.007 \\
\hline 13. BrandTVSpend & 1.21 & .093 & .027 & .036 & .071 & .038 & .036 & -.006 & -.057 & -.003 & .258 & .232 & .056 & .917 & .305 & .003 & -.006 \\
\hline 14. BrandPrintSpend & 1.19 & .116 & .014 & .071 & .045 & .026 & .021 & .002 & -.061 & -.008 & .165 & .152 & .031 & .324 & .143 & -.018 & -.040 \\
\hline 15. CategoryTVSpend & 1.40 & -.092 & -.002 & -.056 & -.011 & .115 & .126 & -.010 & .110 & .449 & -.131 & .028 & .010 & -.002 & -.023 & .963 & .295 \\
\hline 16. CategoryPrintSpend & 1.16 & -.044 & -.006 & -.030 & -.012 & .078 & -.011 & -.003 & .076 & .250 & -.064 & .029 & -.008 & -.001 & -.041 & .329 & .013 \\
\hline
\end{tabular}


Table 6

Focal Product Spillover Effects

\begin{tabular}{|c|c|c|c|c|c|c|c|c|}
\hline & \multicolumn{4}{|c|}{ FocalNonseedSpecialist } & \multicolumn{4}{|c|}{ FocalNonseedGeneralist } \\
\hline & Est. & SE & $\mathbf{p}$ & & Est. & SE & $\mathbf{p}$ & \\
\hline Intercept & -.005 & .007 & .536 & & -.004 & .004 & .247 & \\
\hline FocalSeedSpecialist & .041 & .004 & .000 & $* * *$ & .005 & .003 & .046 & $* *$ \\
\hline FocalNonseedSpecialist & .272 & .003 & .000 & $* * *$ & .015 & .003 & .000 & $* * *$ \\
\hline FocalNonseedGeneralist & .050 & .007 & .000 & $* * *$ & .284 & .002 & .000 & $* * *$ \\
\hline BrandNonseedSpecialist & .005 & .007 & .417 & & .000 & .005 & .934 & \\
\hline BrandNonseedGeneralist & -.002 & .011 & .837 & & .009 & .005 & .076 & $*$ \\
\hline CategoryNonseedSpecialist & .000 & .008 & .964 & & .000 & .005 & .999 & \\
\hline CategoryNonseedGeneralist & -.011 & .005 & .025 & $* *$ & .002 & .003 & .464 & \\
\hline BrandSeedSpecialist & -.004 & .010 & .716 & & .008 & .008 & .279 & \\
\hline CategorySeedSpecialist & .013 & .006 & .037 & $* *$ & .002 & .004 & .524 & \\
\hline SearchTrends & .053 & .005 & .000 & $* * *$ & .020 & .003 & .000 & $* * *$ \\
\hline FocalTVSpend & .006 & .002 & .012 & $* *$ & -.002 & .002 & .313 & \\
\hline FocalPrintSpend & .012 & .002 & .000 & $* * *$ & .000 & .001 & .755 & \\
\hline BrandTVSpend & .000 & .001 & .665 & & .000 & .001 & .557 & \\
\hline BrandPrintSpend & .002 & .001 & .026 & $* *$ & .001 & .000 & .030 & $* *$ \\
\hline CategoryTVSpend & .003 & .001 & .001 & $* * *$ & .000 & .001 & .646 & \\
\hline CategoryPrintSpend & .000 & .001 & .694 & & .000 & .000 & .205 & \\
\hline AvgWords & .000 & .000 & .048 & $* *$ & .000 & .000 & .658 & \\
\hline AvgImages & .003 & .002 & .116 & & .000 & .002 & .829 & \\
\hline TopReviewer & .001 & .003 & .833 & & .002 & .002 & .307 & \\
\hline CategorySMC1 & .005 & .013 & .699 & & -.008 & .008 & .279 & \\
\hline CategorySMC2 & -.009 & .015 & .532 & & .029 & .009 & .002 & $* * *$ \\
\hline CategorySMC3 & .006 & .012 & .621 & & -.016 & .007 & .020 & $* *$ \\
\hline
\end{tabular}

${ }^{*} p<.10,{ }^{* *} p<.05,{ }^{* * *} p<.01$.

Explanatory variables are lagged (except AvgWords, AvgImages, TopReviewer, CategorySMC1/2/3).

$\mathrm{N}=28,654$ over 390 campaigns featuring 192 brands and 11 product categories. 
Table 7

Brand Spillover Effects

\begin{tabular}{|c|c|c|c|c|c|c|c|c|}
\hline & \multicolumn{4}{|c|}{ BrandNonseedSpecialist } & \multicolumn{4}{|c|}{ BrandNonseedGeneralist } \\
\hline & Est. & SE & $\mathbf{p}$ & & Est. & SE & $\mathbf{p}$ & \\
\hline Intercept & -.002 & .007 & .804 & & -.005 & .004 & .278 & \\
\hline FocalSeedSpecialist & -.004 & .004 & .366 & & -.007 & .003 & .012 & $* *$ \\
\hline FocalNonseedSpecialist & .008 & .005 & .097 & $*$ & -.001 & .003 & .748 & \\
\hline FocalNonseedGeneralist & -.002 & .008 & .777 & & .012 & .004 & .001 & $* * *$ \\
\hline BrandNonseedSpecialist & .189 & .004 & .000 & $* * *$ & .030 & .003 & .000 & $* * *$ \\
\hline BrandNonseedGeneralist & .040 & .006 & .000 & $* * *$ & .133 & .003 & .000 & $* * *$ \\
\hline CategoryNonseedSpecialist & .010 & .006 & .118 & & .001 & .004 & .726 & \\
\hline CategoryNonseedGeneralist & .000 & .004 & .916 & & .000 & .002 & .938 & \\
\hline BrandSeedSpecialist & .052 & .006 & .000 & $* * *$ & .003 & .004 & .510 & \\
\hline CategorySeedSpecialist & -.001 & .005 & .914 & & -.007 & .003 & .020 & $* *$ \\
\hline SearchTrends & .011 & .005 & .023 & $* *$ & .007 & .003 & .021 & $* *$ \\
\hline FocalTVSpend & -.001 & .004 & .818 & & -.001 & .005 & .790 & \\
\hline FocalPrintSpend & .002 & .002 & .464 & & .003 & .001 & .013 & $* *$ \\
\hline BrandTVSpend & -.003 & .001 & .013 & $* *$ & -.003 & .001 & .000 & $* * *$ \\
\hline BrandPrintSpend & .002 & .001 & .003 & $* * *$ & .001 & .000 & .157 & \\
\hline CategoryTVSpend & .001 & .001 & .070 & $*$ & .001 & .001 & .154 & \\
\hline CategoryPrintSpend & -.001 & .000 & .029 & $* *$ & -.001 & .000 & .003 & $* * *$ \\
\hline AvgWords & .000 & .000 & .626 & & .000 & .000 & .177 & \\
\hline AvgImages & .001 & .002 & .778 & & .000 & .002 & .750 & \\
\hline TopReviewer & .000 & .004 & .923 & & .000 & .004 & .981 & \\
\hline CategorySMC1 & .001 & .013 & .919 & & .009 & .008 & .229 & \\
\hline CategorySMC2 & -.013 & .016 & .425 & & .000 & .009 & .999 & \\
\hline CategorySMC3 & .014 & .013 & .260 & & -.004 & .007 & .562 & \\
\hline
\end{tabular}

${ }^{*} p<.10,{ }^{* *} p<.05,{ }^{* * *} p<.01$.

Explanatory variables are lagged (except AvgWords, AvgImages, TopReviewer, CategorySMC1/2/3).

$\mathrm{N}=28,654$ over 390 campaigns featuring 192 brands and 11 product categories. 
Table 8

Category Spillover Effects

\begin{tabular}{|c|c|c|c|c|c|c|c|c|}
\hline & \multicolumn{4}{|c|}{ CategoryNonseedSpecialist } & \multicolumn{4}{|c|}{ CategoryNonseedGeneralist } \\
\hline & Est. & $\mathbf{S E}$ & $\mathbf{p}$ & & Est. & SE & $\mathbf{p}$ & \\
\hline Intercept & .011 & .008 & .201 & & -.083 & .013 & .000 & $* * *$ \\
\hline FocalSeedSpecialist & -.012 & .004 & .003 & $* * *$ & -.010 & .006 & .138 & \\
\hline FocalNonseedSpecialist & .001 & .005 & .880 & & -.012 & .008 & .131 & \\
\hline FocalNonseedGeneralist & -.010 & .008 & .198 & & -.002 & .014 & .910 & \\
\hline BrandNonseedSpecialist & -.014 & .006 & .014 & ** & .006 & .009 & .549 & \\
\hline BrandNonseedGeneralist & .000 & .009 & .978 & & -.008 & .015 & .594 & \\
\hline CategoryNonseedSpecialist & .314 & .004 & .000 & **** & .137 & .008 & .000 & $* * *$ \\
\hline CategoryNonseedGeneralist & .044 & .003 & .000 & $* * *$ & .344 & .005 & .000 & $* * *$ \\
\hline BrandSeedSpecialist & .019 & .008 & .019 & $* *$ & .011 & .013 & .368 & \\
\hline CategorySeedSpecialist & .151 & .005 & .000 & **** & .052 & .008 & .000 & $* * *$ \\
\hline SearchTrends & .007 & .006 & .207 & & -.007 & .010 & .492 & \\
\hline FocalTVSpend & -.006 & .002 & .008 & $* * *$ & -.009 & .005 & .094 & $*$ \\
\hline FocalPrintSpend & .000 & .002 & .983 & & .000 & .004 & .949 & \\
\hline BrandTVSpend & .001 & .001 & .608 & & -.002 & .002 & .165 & \\
\hline BrandPrintSpend & -.001 & .001 & .107 & & -.002 & .001 & .218 & \\
\hline CategoryTVSpend & .004 & .001 & .000 & $* * *$ & -.003 & .001 & .000 & $* * *$ \\
\hline CategoryPrintSpend & .001 & .000 & .101 & & -.001 & .001 & .185 & \\
\hline AvgWords & .000 & .000 & .347 & & .000 & .000 & .725 & \\
\hline AvgImages & .001 & .002 & .689 & & .001 & .004 & .855 & \\
\hline TopReviewer & .001 & .003 & .869 & & .001 & .007 & .934 & \\
\hline CategorySMC1 & -.052 & .012 & .000 & $* * *$ & .062 & .020 & .002 & $* * *$ \\
\hline CategorySMC2 & -.049 & .014 & .001 & $* * *$ & .004 & .026 & .873 & \\
\hline CategorySMC3 & .092 & .011 & .000 & $* * *$ & .026 & .021 & .205 & \\
\hline
\end{tabular}

${ }^{*} p<.10,{ }^{* *} p<.05,{ }^{* * *} p<.01$.

Explanatory variables are lagged (except AvgWords, AvgImages, TopReviewer, CategorySMC1/2/3).

$\mathrm{N}=28,654$ over 390 campaigns featuring 192 brands and 11 product categories. 


\section{Web Appendix}

Table WA1

Effects for Focal Product WOM by Seeds

\begin{tabular}{|c|c|c|c|c|}
\hline & \multicolumn{4}{|c|}{ FocalSeedSpecialist } \\
\hline & Est. & SE & p & \\
\hline Intercept & -.035 & .006 & .000 & $* * *$ \\
\hline FocalSeedSpecialist & .188 & .001 & .000 & $* * *$ \\
\hline FocalNonseedSpecialist & .009 & .003 & .005 & $* * *$ \\
\hline FocalNonseedGeneralist & -.007 & .005 & .182 & \\
\hline BrandNonseedSpecialist & -.002 & .006 & .724 & \\
\hline BrandNonseedGeneralist & -.007 & .009 & .425 & \\
\hline CategoryNonseedSpecialist & .002 & .006 & .737 & \\
\hline CategoryNonseedGeneralist & .000 & .004 & .928 & \\
\hline BrandSeedSpecialist & -.004 & .007 & .613 & \\
\hline CategorySeedSpecialist & -.020 & .005 & .000 & $* * *$ \\
\hline SearchTrends & .002 & .005 & .691 & \\
\hline FocalTVSpend & .002 & .001 & .081 & $*$ \\
\hline FocalPrintSpend & .005 & .001 & .000 & $* * *$ \\
\hline BrandTVSpend & -.001 & .001 & .168 & \\
\hline BrandPrintSpend & -.001 & .001 & .239 & \\
\hline CategoryTVSpend & .000 & .001 & .728 & \\
\hline CategoryPrintSpend & .001 & .000 & .104 & \\
\hline AvgWords & .003 & .000 & .000 & $* * *$ \\
\hline AvgImages & .067 & .000 & .000 & $* * *$ \\
\hline TopReviewer & .034 & .000 & .000 & $* * *$ \\
\hline CategorySMC1 & -.007 & .008 & .413 & \\
\hline CategorySMC2 & -.009 & .009 & .310 & \\
\hline CategorySMC3 & .015 & .008 & .056 & $*$ \\
\hline
\end{tabular}


Table WA2

Effects for Same Brand and Same Category WOM by Seeds

\begin{tabular}{|c|c|c|c|c|c|c|c|c|}
\hline & \multicolumn{4}{|c|}{ BrandSeedSpecialist } & \multicolumn{4}{|c|}{ CategorySeedSpecialist } \\
\hline & Est. & SE & $\mathbf{p}$ & & Est. & SE & $\mathbf{p}$ & \\
\hline Intercept & .002 & .003 & .578 & & .032 & .005 & .000 & $* * *$ \\
\hline FocalSeedSpecialist & .003 & .001 & .073 & $*$ & -.008 & .003 & .003 & $* * *$ \\
\hline FocalNonseedSpecialist & .005 & .002 & .006 & $* * *$ & .000 & .003 & .885 & \\
\hline FocalNonseedGeneralist & .000 & .003 & .926 & & .001 & .005 & .802 & \\
\hline BrandNonseedSpecialist & .001 & .002 & .505 & & .000 & .004 & .987 & \\
\hline BrandNonseedGeneralist & -.006 & .003 & .017 & $* *$ & -.001 & .006 & .832 & \\
\hline CategoryNonseedSpecialist & .006 & .002 & .012 & $* *$ & .025 & .005 & .000 & $* * *$ \\
\hline CategoryNonseedGeneralist & .002 & .001 & .254 & & .004 & .003 & .081 & $*$ \\
\hline BrandSeedSpecialist & .844 & .002 & .000 & $* * *$ & .009 & .006 & .123 & \\
\hline CategorySeedSpecialist & .007 & .002 & .000 & $* * *$ & .878 & .004 & .000 & $* * *$ \\
\hline SearchTrends & .003 & .002 & .138 & & .001 & .003 & .791 & \\
\hline FocalTVSpend & .001 & .001 & .257 & & .002 & .003 & .430 & \\
\hline FocalPrintSpend & .001 & .001 & .120 & & .000 & .001 & .733 & \\
\hline BrandTVSpend & .000 & .001 & .529 & & .001 & .001 & .036 & $* *$ \\
\hline BrandPrintSpend & .001 & .000 & .001 & $* * *$ & .001 & .000 & .205 & \\
\hline CategoryTVSpend & .000 & .000 & .744 & & .001 & .001 & .222 & \\
\hline CategoryPrintSpend & .000 & .000 & .222 & & -.001 & .000 & .044 & $* *$ \\
\hline AvgWords & .000 & .000 & .885 & & .000 & .000 & .082 & $*$ \\
\hline AvgImages & -.001 & .001 & .493 & & .003 & .002 & .162 & \\
\hline TopReviewer & .000 & .001 & .806 & & .000 & .003 & .950 & \\
\hline CategorySMC1 & .001 & .005 & .868 & & -.077 & .006 & .000 & $* * *$ \\
\hline CategorySMC2 & -.002 & .006 & .738 & & .068 & .009 & .000 & $* * *$ \\
\hline CategorySMC3 & .001 & .005 & .866 & & -.021 & .008 & .007 & $* * *$ \\
\hline
\end{tabular}

${ }^{*} p<.10,{ }^{* *} p<.05,{ }^{* * *} p<.01$.

Explanatory variables are lagged (except AvgWords, AvgImages, TopReviewer, CategorySMC1/2/3).

$\mathrm{N}=28,654$ over 390 campaigns featuring 192 brands and 11 product categories. 
Table WA3

Effects for Search Trends

\begin{tabular}{|c|c|c|c|c|}
\hline & \multicolumn{4}{|c|}{ SearchTrends } \\
\hline & Est. & SE & $\mathbf{p}$ & \\
\hline Intercept & -.012 & .005 & .032 & $* *$ \\
\hline FocalSeedSpecialist & .001 & .005 & .774 & \\
\hline FocalNonseedSpecialist & .034 & .004 & .000 & $* * *$ \\
\hline FocalNonseedGeneralist & .034 & .006 & .000 & $* * *$ \\
\hline BrandNonseedSpecialist & .005 & .005 & .401 & \\
\hline BrandNonseedGeneralist & .010 & .008 & .194 & \\
\hline CategoryNonseedSpecialist & .005 & .008 & .516 & \\
\hline CategoryNonseedGeneralist & .000 & .004 & .951 & \\
\hline BrandSeedSpecialist & -.001 & .009 & .886 & \\
\hline CategorySeedSpecialist & -.008 & .005 & .119 & \\
\hline SearchTrends & .606 & .002 & .000 & $* * *$ \\
\hline FocalTVSpend & .013 & .003 & .000 & $* * *$ \\
\hline FocalPrintSpend & -.001 & .002 & .600 & \\
\hline BrandTVSpend & -.001 & .001 & .063 & $*$ \\
\hline BrandPrintSpend & .001 & .001 & .072 & $*$ \\
\hline CategoryTVSpend & .001 & .001 & .224 & \\
\hline CategoryPrintSpend & -.001 & .000 & .009 & $* * *$ \\
\hline AvgWords & .000 & .000 & .400 & \\
\hline AvgImages & .001 & .003 & .635 & \\
\hline TopReviewer & .000 & .005 & .996 & \\
\hline CategorySMC1 & -.005 & .009 & .599 & \\
\hline CategorySMC2 & .028 & .010 & .007 & $* * *$ \\
\hline CategorySMC3 & -.011 & .008 & .155 & \\
\hline
\end{tabular}


Table WA4

Effects for Focal Product Advertising

\begin{tabular}{|c|c|c|c|c|c|c|c|c|}
\hline & \multicolumn{4}{|c|}{ FocalTVSpend } & \multicolumn{4}{|c|}{ FocalPrintSpend } \\
\hline & Est. & SE & $\mathbf{p}$ & & Est. & SE & $\mathbf{p}$ & \\
\hline Intercept & .002 & .014 & .892 & & .009 & .023 & .709 & \\
\hline FocalSeedSpecialist & .004 & .005 & .373 & & .024 & .007 & .000 & $* * *$ \\
\hline FocalNonseedSpecialist & .015 & .007 & .024 & $* *$ & .091 & .010 & .000 & $* * *$ \\
\hline FocalNonseedGeneralist & -.002 & .023 & .946 & & .036 & .018 & .042 & $* *$ \\
\hline BrandNonseedSpecialist & .004 & .013 & .743 & & -.035 & .019 & .076 & $*$ \\
\hline BrandNonseedGeneralist & -.001 & .048 & .981 & & .055 & .027 & .043 & $* *$ \\
\hline CategoryNonseedSpecialist & -.002 & .009 & .790 & & .010 & .021 & .627 & \\
\hline CategoryNonseedGeneralist & -.003 & .009 & .703 & & -.011 & .013 & .361 & \\
\hline BrandSeedSpecialist & -.001 & .030 & .961 & & -.020 & .030 & .509 & \\
\hline CategorySeedSpecialist & .002 & .016 & .906 & & -.032 & .017 & .050 & $*$ \\
\hline SearchTrends & -.007 & .009 & .430 & & -.046 & .014 & .001 & $* * *$ \\
\hline FocalTVSpend & .930 & .001 & .000 & $* * *$ & .108 & .003 & .000 & $* * *$ \\
\hline FocalPrintSpend & -.009 & .001 & .000 & $* * *$ & .039 & .002 & .000 & $* * *$ \\
\hline BrandTVSpend & .001 & .002 & .483 & & .002 & .002 & .453 & \\
\hline BrandPrintSpend & -.002 & .001 & .003 & $* * *$ & -.021 & .002 & .000 & $* * *$ \\
\hline CategoryTVSpend & .001 & .002 & .550 & & .004 & .002 & .082 & $*$ \\
\hline CategoryPrintSpend & .000 & .002 & .894 & & .000 & .002 & .876 & \\
\hline AvgWords & .000 & .000 & .987 & & .001 & .000 & .000 & $* * *$ \\
\hline AvgImages & .001 & .003 & .805 & & -.006 & .004 & .162 & \\
\hline TopReviewer & .000 & .007 & .985 & & -.002 & .013 & .873 & \\
\hline CategorySMC1 & -.003 & .095 & .977 & & .003 & .053 & .960 & \\
\hline CategorySMC2 & .000 & .116 & 1.000 & & -.009 & .063 & .890 & \\
\hline CategorySMC3 & -.001 & .074 & .993 & & -.007 & .041 & .870 & \\
\hline
\end{tabular}

${ }^{*} p<.10,{ }^{* *} p<.05,{ }^{* * *} p<.01$.

Explanatory variables are lagged (except AvgWords, AvgImages, TopReviewer, CategorySMC1/2/3).

$\mathrm{N}=28,654$ over 390 campaigns featuring 192 brands and 11 product categories. 
Table WA5

Effects for Brand Advertising

\begin{tabular}{|c|c|c|c|c|c|c|c|c|}
\hline & \multicolumn{4}{|c|}{ BrandTVSpend } & \multicolumn{4}{|c|}{ BrandPrintSpend } \\
\hline & Est. & SE & $\mathbf{p}$ & & Est. & SE & $\mathbf{p}$ & \\
\hline Intercept & .020 & .017 & .249 & & .028 & .050 & .580 & \\
\hline FocalSeedSpecialist & -.024 & .012 & .044 & $* *$ & -.031 & .032 & .339 & \\
\hline FocalNonseedSpecialist & -.020 & .012 & .111 & & .086 & .028 & .002 & $* * *$ \\
\hline FocalNonseedGeneralist & -.006 & .019 & .738 & & .035 & .049 & .476 & \\
\hline BrandNonseedSpecialist & .003 & .025 & .900 & & -.029 & .050 & .555 & \\
\hline BrandNonseedGeneralist & -.019 & .029 & .521 & & .150 & .066 & .024 & $* *$ \\
\hline CategoryNonseedSpecialist & -.003 & .023 & .905 & & -.010 & .059 & .867 & \\
\hline CategoryNonseedGeneralist & .000 & .012 & .995 & & .007 & .034 & .838 & \\
\hline BrandSeedSpecialist & -.064 & .049 & .189 & & -.051 & .077 & .509 & \\
\hline CategorySeedSpecialist & -.025 & .016 & .116 & & -.123 & .041 & .003 & $* * *$ \\
\hline SearchTrends & -.016 & .010 & .121 & & -.029 & .030 & .338 & \\
\hline FocalTVSpend & -.005 & .005 & .314 & & -.167 & .015 & .000 & $* * *$ \\
\hline FocalPrintSpend & -.019 & .004 & .000 & $* * *$ & -.170 & .014 & .000 & $* * *$ \\
\hline BrandTVSpend & .876 & .002 & .000 & $* * *$ & .083 & .004 & .000 & $* * *$ \\
\hline BrandPrintSpend & -.012 & .001 & .000 & $* * *$ & -.021 & .004 & .000 & $* * *$ \\
\hline CategoryTVSpend & -.002 & .002 & .349 & & .018 & .006 & .006 & $* * *$ \\
\hline CategoryPrintSpend & -.001 & .002 & .695 & & -.050 & .004 & .000 & $* * *$ \\
\hline AvgWords & .000 & .000 & .285 & & .000 & .001 & .761 & \\
\hline AvgImages & .005 & .010 & .623 & & .005 & .022 & .825 & \\
\hline TopReviewer & .000 & .026 & .998 & & -.004 & .022 & .856 & \\
\hline CategorySMC1 & -.129 & .034 & .000 & $* * *$ & -.127 & .077 & .099 & $*$ \\
\hline CategorySMC2 & .053 & .042 & .205 & & .406 & .088 & .000 & $* * *$ \\
\hline CategorySMC3 & .057 & .029 & .046 & $* *$ & -.310 & .068 & .000 & $* * *$ \\
\hline
\end{tabular}

${ }^{*} p<.10,{ }^{* *} p<.05,{ }^{* * *} p<.01$.

Explanatory variables are lagged (except AvgWords, AvgImages, TopReviewer, CategorySMC1/2/3).

$\mathrm{N}=28,654$ over 390 campaigns featuring 192 brands and 11 product categories. 
Table WA6

Effects for Category Advertising

\begin{tabular}{lcccccccc}
\hline & \multicolumn{3}{c}{ CategoryTVSpend } & \multicolumn{3}{c}{ CategoryPrintSpend } \\
& Est. & SE & p & & Est. & SE & p & \\
\hline Intercept & -.092 & .017 & .000 & $* * *$ & .195 & .080 & .015 & $* *$ \\
FocalSeedSpecialist & .008 & .021 & .703 & & -.052 & .061 & .397 & \\
FocalNonseedSpecialist & -.009 & .017 & .619 & & .157 & .063 & .013 & $* *$ \\
FocalNonseedGeneralist & -.001 & .028 & .959 & & -.060 & .095 & .530 & \\
BrandNonseedSpecialist & .015 & .024 & .520 & & -.016 & .081 & .848 & \\
BrandNonseedGeneralist & -.013 & .046 & .769 & & .088 & .132 & .503 & \\
CategoryNonseedSpecialist & -.052 & .021 & .013 & $* *$ & .161 & .100 & .108 & \\
CategoryNonseedGeneralist & .028 & .014 & .054 & $*$ & .145 & .057 & .011 & $* *$ \\
BrandSeedSpecialist & -.002 & .036 & .954 & & .202 & .119 & .088 & $*$ \\
CategorySeedSpecialist & -.092 & .024 & .000 & $* * *$ & -.762 & .073 & .000 & $* * *$ \\
SearchTrends & .060 & .026 & .020 & $* *$ & -.161 & .056 & .004 & $* * *$ \\
FocalTVSpend & -.011 & .008 & .190 & & .053 & .068 & .434 & \\
FocalPrintSpend & -.017 & .007 & .012 & $* *$ & -.050 & .040 & .204 & \\
BrandTVSpend & .002 & .004 & .551 & & .016 & .014 & .251 & \\
BrandPrintSpend & -.011 & .003 & .000 & $* * *$ & -.094 & .011 & .000 & $* * *$ \\
CategoryTVSpend & .910 & .003 & .000 & $* * *$ & .129 & .009 & .000 & $* * *$ \\
CategoryPrintSpend & -.008 & .002 & .000 & $* * *$ & -.009 & .005 & .091 & $*$ \\
AvgWords & -.001 & .001 & .202 & & -.004 & .002 & .047 & $* *$ \\
AvgImages & .012 & .014 & .392 & & .070 & .037 & .057 & $*$ \\
TopReviewer & .000 & .028 & .996 & & -.009 & .049 & .860 & \\
CategorySMC1 & .090 & .085 & .292 & & -.302 & .140 & .031 & $* *$ \\
CategorySMC2 & .028 & .103 & .785 & & .961 & .156 & .000 & $* * *$ \\
CategorySMC3 & -.033 & .056 & .560 & & -.884 & .121 & .000 & $* * *$ \\
\hline$* p<10 * * p<05, p<01$ & & & & & & & &
\end{tabular}

Explanatory variables are lagged (except AvgWords, AvgImages, TopReviewer, CategorySMC1/2/3).

$\mathrm{N}=28,654$ over 390 campaigns featuring 192 brands and 11 product categories. 
Table WA7

Error Correlations

\begin{tabular}{|c|c|c|c|c|c|c|c|c|c|c|c|c|c|c|c|}
\hline & $\mathbf{1}$ & 2 & 3 & 4 & 5 & 6 & 7 & 8 & 9 & 10 & 11 & 12 & 13 & 14 & 15 \\
\hline 1. FocalNonseedSpecialist & & & & & & & & & & & & & & & \\
\hline 2. FocalNonseedGeneralist & .034 & & & & & & & & & & & & & & \\
\hline 3. BrandNonseedSpecialist & .108 & .012 & & & & & & & & & & & & & \\
\hline 4. BrandNonseedGeneralist & .017 & .076 & .065 & & & & & & & & & & & & \\
\hline 5. CategoryNonseedSpecialist & .027 & -.001 & .081 & .017 & & & & & & & & & & & \\
\hline 6. CategoryNonseedGeneralist & -.006 & .026 & .026 & .078 & .122 & & & & & & & & & & \\
\hline 7. FocalSeedSpecialist & .024 & .002 & -.003 & .000 & -.002 & .011 & & & & & & & & & \\
\hline 8. BrandSeedSpecialist & .009 & .004 & .033 & .048 & .019 & .007 & -.008 & & & & & & & & \\
\hline 9. CategorySeedSpecialist & -.007 & .003 & .011 & -.004 & .087 & .069 & .004 & .057 & & & & & & & \\
\hline 10. SearchTrends & .032 & .027 & -.011 & -.001 & .007 & .001 & .001 & .007 & .014 & & & & & & \\
\hline 11. FocalTVSpend & .008 & .001 & .005 & .000 & .004 & -.001 & .020 & -.008 & .001 & .011 & & & & & \\
\hline 12. FocalPrintSpend & .026 & -.002 & -.013 & .002 & .000 & -.016 & -.016 & -.006 & -.004 & -.001 & .078 & & & & \\
\hline 13. BrandTVSpend & -.006 & -.017 & -.013 & -.011 & -.002 & .008 & -.006 & -.007 & .006 & .004 & .048 & -.012 & & & \\
\hline 14. BrandPrintSpend & -.013 & -.008 & -.002 & -.002 & -.009 & -.029 & .001 & .005 & -.015 & .007 & .008 & .129 & .030 & & \\
\hline 15. CategoryTVSpend & -.001 & .010 & .001 & .003 & -.004 & .015 & .002 & -.011 & -.028 & .009 & .032 & .015 & .009 & .018 & \\
\hline 16. CategoryPrintSpend & -.016 & -.032 & -.007 & -.027 & -.052 & -.084 & -.005 & .002 & .005 & .001 & .013 & .062 & .007 & .250 & .050 \\
\hline
\end{tabular}

